October 26, 2018

\title{
Integrable and Conformal Boundary Conditions for $\mathbb{Z}_{k}$ Parafermions on a Cylinder
}

\author{
Christian Mercat and Paul A. Pearce \\ Department of Mathematics and Statistics \\ University of Melbourne \\ Parkville, Victoria 3010, Australia
}

\begin{abstract}
We study integrable and conformal boundary conditions for $\hat{s \ell}(2) \mathbb{Z}_{k}$ parafermions on a cylinder. These conformal field theories are realized as the continuum scaling limit of critical $A-D-E$ lattice models with negative spectral parameter. The conformal boundary conditions labelled by $(a, m) \in\left(G, \mathbb{Z}_{2 k}\right)$ are identified with associated integrable lattice boundary conditions labelled by $(r, a) \in\left(A_{g-2}, G\right)$ where $g$ is the Coxeter number of the $A-D-E$ graph $G$. We obtain analytically the boundary free energies, present general expressions for the parafermion cylinder partition functions and confirm these results by numerical calculations.
\end{abstract}

\section{Introduction}

There has been much recent progress [1, 2, 国, 因, 5, 6, 团 on understanding integrable boundaries in statistical mechanics, conformal boundary conditions in rational conformal field theories and the intimate relations between them. Indeed it appears that, for certain classes of theories, all of the conformal boundary conditions can be realized as the continuum scaling limit of integrable boundary conditions for the associated integrable lattice models. For simple rational conformal field theories, such as $\hat{s} \ell(2)$ minimal theories, a complete classification has been given [1, 2, 国] of the conformal boundary conditions. These are labelled by nodes $(r, a)$ of a tensor product graph $A \otimes G$ where the pair of graphs $(A, G)$, with $G$ of $A-D-E$ type, coincide precisely with the pairs in the $A-D-E$ classification of Cappelli, Itzykson and Zuber [8]. Moreover, for the unitary minimal models the physical content of the boundary conditions has been ascertained [1] by studying the related integrable boundary conditions of the associated $A-D-E$ lattice models [9] with positive spectral parameter $u>0$.

It is highly desirable to carry out similar studies on boundary conditions for other classes of conformal field theories and their associated lattice models such as the superconformal and higher fusion models, higher rank models and so on. In this paper we study the boundary 
conditions of $\mathbb{Z}_{k}$ parafermionic models [10, 11, 12, 13, 14, 15, 16, 17, 18, 19]. This class of rational conformal field theories admits an $A-D-E$ classification [11, 15] similar to the classification of Cappelli, Itzykson and Zuber and is associated to the same $A-D$ - $E$ lattice models but in the regime with negative spectral parameter $u<0$. The first two members of the $A$ series are the Ising model $\left(A_{3}\right)$ and the hard hexagon model [20] $\left(A_{4}\right)$ which has the same central charge as the 3 -state Potts model but admits the $W_{3}$ algebra as the extended chiral algebra and has 6 rather than 8 conformal boundary conditions.

The layout of the paper is as follows. In Section 2, we review the conformal properties of $\hat{s \ell}(2) \mathbb{Z}_{k}$ parafermions, their central charges, conformal weights, characters, Kac tables and modular matrices. We also discuss the modular invariant partition functions on the torus and the cylinder partition functions. In Section 3, we present the lattice realizations of these theories. We define the $A-D$ - $E$ lattice models and their integrable boundary weights, present their finite size corrections and compute their bulk and boundary free energies. In Section $⿴$ we identify, through numerical analysis, the integrable boundary conditions from the lattice model with the conformal boundary conditions of parafermions. In Section 5 , we discuss our numerical techniques and present some typical numerical data on which our results are based. We conclude in Section 6 with a short discussion.

\section{$2 \quad \mathbb{Z}_{k}$ Parafermions}

In this section we review the conformal properties of $\hat{s \ell}(2) \mathbb{Z}_{k}$ parafermions. In particular, we review the $A-D-E$ classifications [11, 15] of torus partition functions and present general expressions for the cylinder partition functions following the general framework of Behrend, Pearce, Petkova and Zuber [3] on the assumption that the conformal boundary conditions are labelled by nodes of the tensor product graph

$$
G \otimes \mathbb{Z}_{2 k}
$$

where the Coxeter number of $G$ is $g=k+2$ and $\mathbb{Z}_{2 k}$ denotes the oriented cyclic graph with $2 k$ nodes.

\subsection{Conformal data}

The $\hat{s \ell}(2) \mathbb{Z}_{k}$ parafermionic conformal field theories are $\hat{u}(1)$ cosets of the level $k, \hat{s \ell}(2)$ WZW models

$$
\frac{\hat{s \ell}(2)_{k}}{\hat{u}(1)}
$$

with central charges

$$
c=\frac{3 k}{k+2}-1=\frac{2(k-1)}{k+2}
$$


The conformal weights of primary fields $\phi_{(\ell, m)}$ are given by

$$
\Delta_{(\ell, m)}=\frac{\ell(\ell+2)}{4(k+2)}-\frac{m^{2}}{4 k}, \quad 0 \leq \ell \leq k, \quad 0 \leq|m| \leq \ell, \quad \ell-m \in 2 \mathbb{Z}
$$

and the associated level $k$ characters

$$
\chi_{(\ell, m)}(q)=\eta(q) c_{m}^{\ell}(q)
$$

are given by the string functions 15

$$
\begin{aligned}
c_{m}^{\ell}(q)= & \frac{q^{-\frac{c-2}{24}+\Delta_{(\ell, m)}}}{\eta^{3}(q)} \sum_{r, s=0}^{\infty}(-1)^{r+s} q^{s(s+1) / 2+r(r+1) / 2+r s(k+1)} \\
& \times\left[q^{s(\ell-m) / 2+r(\ell+m) / 2}-q^{k+1-\ell+s(2 k+2-\ell+m) / 2+r(2 k+2-\ell-m) / 2}\right]
\end{aligned}
$$

where $\eta(q)=q^{\frac{1}{24}} \prod_{n=1}^{\infty}\left(1-q^{n}\right)$ is the Dedekind eta function and $q=e^{2 \pi i \tau}$ is the modular parameter.

The conformal weights and characters are extended to the grid

$$
0 \leq \ell \leq k, \quad 0 \leq m \leq 2 k-1, \quad \ell-m \in 2 \mathbb{Z}
$$

by identifying the underlying operators

$$
\phi_{(\ell, m)}=\phi_{(\ell,-m)}^{*}=\phi_{(\ell, m+2 k)}=\phi_{(k-\ell, k-m)}^{*}
$$

where $*$ denotes conjugation. The conformal weights $\Delta_{(\ell, m)}$ and characters $\chi_{(\ell, m)}(q)$ are of course not effected by conjugation. The parafermionic "Kac tables" for the critical Ising $\left(G=A_{3}, k=2\right)$, critical 3-state Potts $\left(G=A_{4}, k=3\right)$ and $\left(G=A_{5}, k=4\right)$ models are shown in Table 1. Each operator appears twice in these grids. To remove this redundancy we could fix a fundamental domain to be the left half of the Kac table. But in order to stress the underlying structure of the fusion algebras we will sometimes make other choices, such as the even sub-lattice with $\ell$ and $m$ even.

Under modular transformations, the level $k$ parafermionic characters transform as

$$
\chi_{(\ell, m)}\left(e^{2 \pi i \tau}\right)=\sum_{\left(\ell^{\prime} \cdot m^{\prime}\right)} S_{(\ell, m)}{ }^{\left(\ell^{\prime}, m^{\prime}\right)} \chi_{\left(\ell^{\prime}, m^{\prime}\right)}\left(e^{-2 \pi i / \tau}\right)
$$

where the modular matrix $S$ satisfies

$$
S^{\dagger}=S^{-1}, \quad S^{4}=I, \quad S^{2}=C
$$

and $C$ is the matrix implementing conjugation. If $(\ell, m)$ ranges over the full conformal grid then $S=S_{W Z W} \otimes S_{\mathbb{Z}_{2 k}}$ is just the tensor product of the $\hat{s \ell}(2)_{k}$ Wess-Zumino-Witten modular matrix and the $\hat{s \ell}(2 k)_{1}$ modular matrix. Restricting to a fundamental domain, the entries of the $k(k+1) / 2 \times k(k+1) / 2$ modular matrix are simply renormalized by a factor of 2 and given explicitly by

$$
S_{(\ell, m)}^{\left(\ell^{\prime}, m^{\prime}\right)}=\frac{1}{\sqrt{k(k+2)}} \sin \frac{\pi(\ell+1)\left(\ell^{\prime}+1\right)}{(k+2)} \exp \frac{\pi i m m^{\prime}}{k}
$$



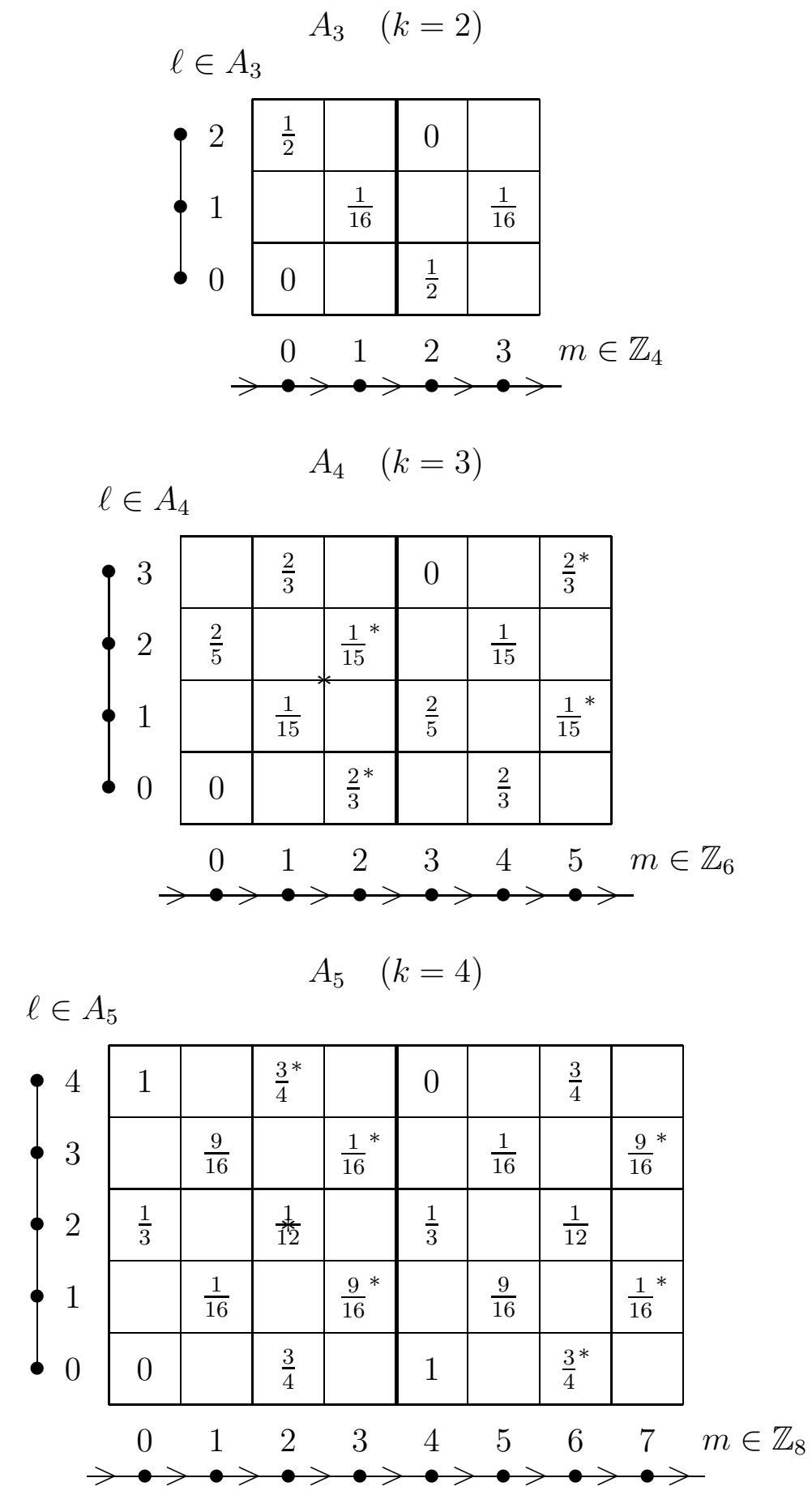

Table 1: Conformal grids of conformal weights for critical Ising $\left(A_{3}, \mathbb{Z}_{4}\right), W_{3}\left(A_{4}, \mathbb{Z}_{6}\right)$ and critical $\left(A_{5}, \mathbb{Z}_{8}\right)$ models. The conjugation $(l, m) \mapsto(k-l, k-m)$ is realized as a central inversion symmetry through the point shown as $*$. 


\section{$2.2 \mathbb{Z}_{k}$ parafermions on a torus}

The $\hat{s \ell}(2) \mathbb{Z}_{k}$ parafermions on a torus have been classified by Gepner and Qiu 11 using modular invariance. They distinguish principal and non-principal cases and their results are summarised in Tables 2 and 3. Here we will be primarily concerned with the principal cases for which the identification with lattice models is clear. We conjecture that non-principal models are associated with fused models [21, 22].

\section{3 $\mathbb{Z}_{k}$ parafermions on a cylinder}

In this section we consider the $\mathbb{Z}_{k}$ parafermion models on a cylinder. We restrict our attention to the principal cases $\left(G, \mathbb{Z}_{2 k}\right)$ where $G$ is of $A, D$ or $E$ type. When $G$ is of $A$ type, the theory is diagonal and the cylinder partition functions are

$$
Z_{\left(\ell_{1}, m_{1}\right) \mid\left(\ell_{2}, m_{2}\right)}(q)=\sum_{(\ell, m)} N_{(\ell, m)\left(\ell_{1}, m_{1}\right)}{ }^{\left(\ell_{2}, m_{2}\right)} \chi_{(\ell, m)}(q)
$$

where $\left(\ell_{1}, m_{1}\right)$ labels the boundary condition on the left, $\left(\ell_{2}, m_{2}\right)$ labels the boundary condition on the right and the fusion coefficients are given by the Verlinde formula [23]

$$
N_{(\ell, m)\left(\ell_{1}, m_{1}\right)}^{\left(\ell_{2}, m_{2}\right)}=\sum_{\left(\ell^{\prime}, m^{\prime}\right)} \frac{S_{(\ell, m)}\left(\ell^{\prime}, m^{\prime}\right) S_{\left(\ell_{1}, m_{1}\right)}{ }^{\left(\ell^{\prime}, m^{\prime}\right)} S_{\left(\ell_{2}, m_{2}\right)}\left(\ell^{\prime}, m^{\prime}\right)^{*}}{S_{(0,0)}\left(\ell^{\prime}, m^{\prime}\right)} \in \mathbb{N}
$$

The parafermionic cylinder partition functions for $\left(A_{4}, \mathbb{Z}_{6}\right)$ are shown in Table 4 . The characters associated to conjugate primary fields, although equal, have not been used so that this table encodes the complete fusion algebra with the conjugate operators shown in the first column.

If $G$ is of $D$ or $E$ type, the boundary conditions are labelled by $(a, m) \in\left(G, \mathbb{Z}_{2 k}\right)$ and the cylinder partition functions are given by [3]:

$$
Z_{\left(a_{1}, m_{1}\right) \mid\left(a_{2}, m_{2}\right)}(q)=\sum_{(\ell, m)} V_{(\ell, m)\left(a_{1}, m_{1}\right)}{ }^{\left(a_{2}, m_{2}\right)} \chi_{(\ell, m)}(q)
$$

where the sum is restricted to a fundamental domain of the Kac table. The intertwiners are

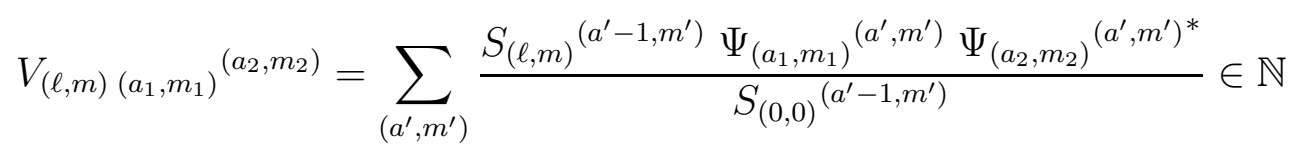

where the sum is over $a^{\prime} \in \operatorname{Exp}(G)$ the Coxeter exponents of $G$ and $m^{\prime} \in \mathbb{Z}_{2 k}$. The eigenvectors of the adjacency matrix of the graph $G \otimes \mathbb{Z}_{2 k}$ are given by the tensor product

$$
\Psi_{(a, m)}\left(a^{\prime}, m^{\prime}\right)=\psi_{a}{ }^{a^{\prime}} \exp \frac{\pi i m m^{\prime}}{k}
$$

where $\psi^{a^{\prime}}$ is the eigenvector, labelled by $a^{\prime} \in \operatorname{Exp}(G)$, of the adjacency matrix $G$. 


$$
\begin{aligned}
& \left(A_{k+1}, \mathbb{Z}_{2 k}\right): \quad \frac{1}{2} \sum_{m=0}^{2 k-1} \sum_{\ell=0}^{k}\left|\chi_{(\ell, m)}\right|^{2} \\
& \left(D_{2 n+2}, \mathbb{Z}_{8 n}\right): \quad \sum_{m=0}^{2 k-1}\left\{\sum_{\substack{\ell=0 \\
\ell \text { even } \\
2 k-1}}^{k / 2-1}\left\{\left|\chi_{(\ell, m)}\right|^{2}+\chi_{(k-\ell, m)} \bar{\chi}_{(\ell, m)}\right\}+2\left|\chi_{(k / 2, m)}\right|^{2}\right\} \\
& (k=4 n) \quad=\frac{1}{2} \sum_{m=0}^{2 k-1} \sum_{\substack{\ell=0 \\
\ell \text { even }}}^{k}\left|\chi_{(\ell, m)}+\chi_{(k-\ell, m)}\right|^{2} \\
& \left(D_{2 n+1}, \mathbb{Z}_{8 n-4}\right): \frac{1}{2} \sum_{m=0}^{2 k-1}\left\{\sum_{\substack{\ell=0 \\
\ell \text { even }}}^{k}\left|\chi_{(\ell, m)}\right|^{2}+\sum_{\substack{\ell=0 \\
\ell \text { odd }}}^{k} \chi_{(k-\ell, m)} \bar{\chi}_{(\ell, m)}\right\} \\
& (k=4 n-2) \\
& \left(E_{6}, \mathbb{Z}_{20}\right): \quad \frac{1}{2} \sum_{m=0}^{2 k-1}\left\{\left|\chi_{(0, m)}+\chi_{(6, m)}\right|^{2}+\left|\chi_{(3, m)}+\chi_{(7, m)}\right|^{2}+\left|\chi_{(4, m)}+\chi_{(10, m)}\right|^{2}\right\} \\
& (k=10) \\
& \left(E_{7}, \mathbb{Z}_{32}\right): \quad \frac{1}{2} \sum_{m=0}^{2 k-1}\left\{\left|\chi_{(0, m)}+\chi_{(16, m)}\right|^{2}+\left|\chi_{(4, m)}+\chi_{(12, m)}\right|^{2}+\left|\chi_{(6, m)}+\chi_{(10, m)}\right|^{2}\right. \\
& \left.(k=16)+\left|\chi_{(8, m)}\right|^{2}+\left(\chi_{(2, m)}+\chi_{(14, m)}\right) \bar{\chi}_{(8, m)}+\chi_{(8, m)}\left(\bar{\chi}_{(2, m)}+\bar{\chi}_{(14, m)}\right)\right\} \\
& \left(E_{8}, \mathbb{Z}_{56}\right): \quad \frac{1}{2} \sum_{m=0}^{2 k-1}\left\{\left|\chi_{(0, m)}+\chi_{(10, m)}+\chi_{(18, m)}+\chi_{(28, m)}\right|^{2}\right. \\
& (k=28) \\
& \left.+\left|\chi_{(6, m)}+\chi_{(12, m)}+\chi_{(16, m)}+\chi_{(22, m)}\right|^{2}\right\}
\end{aligned}
$$

Table 2: The $A-D-E$ classification of modular invariant partition functions for principal $\mathbb{Z}_{k}$ theories. 


$$
\begin{aligned}
& \left(A_{k+1}, \mathbb{Z}_{2 \beta}\right): \quad \frac{1}{4} \sum_{x \in \mathbb{Z}_{2 \beta}, y \in \mathbb{Z}_{2 \alpha}} \sum_{\ell=0}^{k} \chi_{\left(\ell, \alpha^{+}\right)} \bar{\chi}_{\left(\ell, \alpha^{-}\right)} \\
& \left(D_{2 n+2}, \mathbb{Z}_{2 \beta}\right): \frac{1}{8} \sum_{x \in \mathbb{Z}_{2 \beta}, y \in \mathbb{Z}_{2 \alpha} \sum_{\ell=0}} \sum_{\substack{\ell \\
\text { even }}}^{k}\left(\chi_{\left(\ell, \alpha^{+}\right)}+\chi_{\left(k-\ell, \alpha^{+}\right)}\right)\left(\bar{\chi}_{\left(\ell, \alpha^{-}\right)}+\bar{\chi}_{\left(k-\ell, \alpha^{-}\right)}\right) \\
& (k=4 n) \\
& \left(D_{2 n+1}, \mathbb{Z}_{2 \beta}\right): \frac{1}{4} \sum_{x \in \mathbb{Z}_{2 \beta}, y \in \mathbb{Z}_{2 \alpha}}\left\{\sum_{\substack{\ell=0 \\
\ell \text { even }}}^{k} \chi_{\left(\ell, \alpha^{+}\right)} \bar{\chi}_{\left(\ell, \alpha^{-}\right)}+\sum_{\substack{\ell=0 \\
\ell \text { odd }}}^{k} \chi_{\left(k-\ell, \alpha^{+}\right)} \bar{\chi}_{\left(\ell, \alpha^{-}\right)}\right\} \\
& (k=4 n-2) \\
& \left(E_{6}, \mathbb{Z}_{2 \beta}\right): \quad \frac{1}{4} \sum_{x \in \mathbb{Z}_{2 \beta}, y \in \mathbb{Z}_{2 \alpha}}\left\{\left(\chi_{\left(0, \alpha^{+}\right)}+\chi_{\left(6, \alpha^{+}\right)}\right)\left(\bar{\chi}_{\left(0, \alpha^{-}\right)}+\bar{\chi}_{\left(6, \alpha^{-}\right)}\right)\right. \\
& \left.(k=10)+\left(\chi_{\left(3, \alpha^{+}\right)}+\chi_{\left(7, \alpha^{+}\right)}\right)\left(\bar{\chi}_{\left(3, \alpha^{-}\right)}+\bar{\chi}_{\left(7, \alpha^{-}\right)}\right)+\left(\chi_{\left(4, \alpha^{+}\right)}+\chi_{\left(10, \alpha^{+}\right)}\right)\left(\bar{\chi}_{\left(4, \alpha^{-}\right)}+\bar{\chi}_{\left(10, \alpha^{-}\right)}\right)\right\} \\
& \left(E_{7}, \mathbb{Z}_{2 \beta}\right): \quad \frac{1}{4} \sum_{x \in \mathbb{Z}_{2 \beta}, y \in \mathbb{Z}_{2 \alpha}}\left\{\left(\chi_{\left(0, \alpha^{+}\right)}+\chi_{\left(16, \alpha^{+}\right)}\right)\left(\bar{\chi}_{\left(0, \alpha^{-}\right)}+\bar{\chi}_{\left(16, \alpha^{-}\right)}\right)\right. \\
& +\left(\chi_{\left(4, \alpha^{+}\right)}+\chi_{\left(12, \alpha^{+}\right)}\right)\left(\bar{\chi}_{\left(4, \alpha^{-}\right)}+\bar{\chi}_{\left(12, \alpha^{-}\right)}\right)+\left(\chi_{\left(6, \alpha^{+}\right)}+\chi_{\left(10, \alpha^{+}\right)}\right)\left(\bar{\chi}_{\left(6, \alpha^{-}\right)}+\bar{\chi}_{\left(10, \alpha^{-}\right)}\right) \\
& \left.(k=16)+\chi_{\left(8, \alpha^{+}\right)} \bar{\chi}_{\left(8, \alpha^{-}\right)}+\left(\chi_{\left(2, \alpha^{+}\right)}+\chi_{\left(14, \alpha^{+}\right)}\right) \bar{\chi}_{\left(8, \alpha^{-}\right)}+\chi_{\left(8, \alpha^{+}\right)}\left(\bar{\chi}_{\left(2, \alpha^{-}\right)}+\bar{\chi}_{\left(14, \alpha^{-}\right)}\right)\right\} \\
& \left(E_{8}, \mathbb{Z}_{2 \beta}\right): \quad \frac{1}{4} \sum_{x \in \mathbb{Z}_{2 \beta}, y \in \mathbb{Z}_{2 \alpha}}\left\{\left(\chi_{\left(0, \alpha^{+}\right)}+\chi_{\left(10, \alpha^{+}\right)}+\chi_{\left(18, \alpha^{+}\right)}+\chi_{\left(28, \alpha^{+}\right)}\right)\left(\bar{\chi}_{\left(0, \alpha^{-}\right)}+\bar{\chi}_{\left(10, \alpha^{-}\right)}+\bar{\chi}_{\left(18, \alpha^{-}\right)}+\bar{\chi}_{\left(28, \alpha^{-}\right)}\right)\right. \\
& (k=28) \\
& \left.+\left(\chi_{\left(6, \alpha^{+}\right)}+\chi_{\left(12, \alpha^{+}\right)}+\chi_{\left(16, \alpha^{+}\right)}+\chi_{\left(22, \alpha^{+}\right)}\right)\left(\bar{\chi}_{\left(6, \alpha^{-}\right)}+\bar{\chi}_{\left(12, \alpha^{-}\right)}+\bar{\chi}_{\left(16, \alpha^{-}\right)}+\bar{\chi}_{\left(22, \alpha^{-}\right)}\right)\right\}
\end{aligned}
$$

Table 3: The $A-D-E$ classification of modular invariant partition functions for non-principal $\mathbb{Z}_{k}$ theories. Here $\alpha \neq 1$ is a divisor of $k=\alpha \beta$ and $\alpha^{+}=\alpha x+\beta y, \alpha^{-}=\alpha x-\beta y$. 


\begin{tabular}{cc|cccccc}
\multicolumn{7}{c}{ Right } \\
\cline { 3 - 8 } Left & $(\ell, m)$ & $(0,0)$ & $(2,0)$ & $(0,2)$ & $(2,2)$ & $(0,4)$ & $(2,4)$ \\
\cline { 2 - 8 } & $(0,0)$ & $\chi_{(0,0)}$ & $\chi_{(0,2)}$ & $\chi_{(0,4)}$ & $\chi_{(2,0)}$ & $\chi_{(2,2)}$ & $\chi_{(2,4)}$ \\
& $(0,2)$ & $\chi_{(0,4)}$ & $\chi_{(0,0)}$ & $\chi_{(0,2)}$ & $\chi_{(2,4)}$ & $\chi_{(2,0)}$ & $\chi_{(2,2)}$ \\
& $(2,0)$ & $\chi_{(0,2)}$ & $\chi_{(0,4)}$ & $\chi_{(0,0)}$ & $\chi_{(2,2)}$ & $\chi_{(2,4)}$ & $\chi_{(2,0)}$ \\
& $(2,2)$ & $\chi_{(2,0)}$ & $\chi_{(2,2)}$ & $\chi_{(2,4)}$ & $\chi_{(0,0)}+\chi_{(2,0)}$ & $\chi_{(0,2)}+\chi_{(2,2)}$ & $\chi_{(0,4)}+\chi_{(2,4)}$ \\
& $(2,4)$ & $\chi_{(2,0)}$ & $\chi_{(2,2)}$ & $\chi_{(0,4)}+\chi_{(2,4)}$ & $\chi_{(0,0)}+\chi_{(2,0)}$ & $\chi_{(0,2)}+\chi_{(2,2)}$ \\
& $\chi_{(2,2)}$ & $\chi_{(2,4)}$, & $\chi_{(2,0)}$ & $\chi_{(0,2)}+\chi_{(2,2)}$ & $\chi_{(0,4)}+\chi_{(2,4)}$ & $\chi_{(0,0)}+\chi_{(2,0)}$
\end{tabular}

Table 4: Cylinder partition functions $Z_{L \mid R}$ for the $W_{3}$ model $\left(A_{4}, \mathbb{Z}_{6}\right) \sim\left(T_{2}, \mathbb{Z}_{3}\right)$.

The intertwiner can alternatively be defined as the tensor product of intertwiners for each graph

$$
V_{(\ell, m)\left(a_{1}, m_{1}\right)}^{\left(a_{2}, m_{2}\right)}=V_{\ell+1, a_{1}{ }^{a_{2}}} V_{m+1, m_{1}}^{Z} m_{2}
$$

where the intertwiners for $G$ and $Z=\mathbb{Z}_{2 k}$ are defined by a formulas similar to (2.15). The intertwiner for the graph $G$ satisfies the recurrence relation

$$
V_{i}^{G}:=V_{2}^{G} \cdot V_{i-1}^{G}-V_{i-2}^{G}
$$

where $V_{1}^{G}=I$ and $V_{2}^{G}=G$, the adjacency matrix of $G$. The intertwiner for $\mathbb{Z}_{2 k}$ is simply a power of the adjacency matrix $V_{m}^{Z}=\left(\mathbb{Z}_{2 k}\right)^{m}$ of the directed graph $\mathbb{Z}_{2 k}$.

The parafermionic cylinder partition functions for $\left(A_{5}, \mathbb{Z}_{8}\right)$ and $\left(D_{4}, \mathbb{Z}_{8}\right)$ are shown in Tables 56. In the $A_{5}$ case the characters associated with conjugate fields have been kept separated in order to encode the complete fusion algebra, but have been identified in the $D_{4}$ case to make the $\mathbb{Z}_{3}$ symmetry apparent when these $\left(D_{4}, \mathbb{Z}_{8}\right)$ partition functions are written in terms of the fundamental string functions.

In the general $D$ and $E$ cases, these partition functions are best understood in terms of extended (block) characters [3]. These extended characters $\hat{\chi}_{(a, m)}$ are linear forms in the $\left(A_{L}, \mathbb{Z}_{2 k}\right)$ characters involving the fundamental intertwiner

$$
\hat{\chi}_{(a, m)}(q):=\sum_{(\ell, n)} V_{(\ell, n)(0,0)}^{(a, m)} \chi_{(\ell, n)}(q)
$$

where the sum is over a fundamental domain. Note that for the $\left(D_{\frac{k}{2}+2}, \mathbb{Z}_{2 k}\right)$ models the conformal dimensions of the two primary fields appearing in the extended characters differ by integers or half integers

$$
\Delta_{(\ell, m)}-\Delta_{(k-\ell, m)}=\frac{\ell(\ell+2)-(k-\ell)(k-\ell+2)}{4(k+2)}=\frac{\ell-\frac{k}{2}}{2}
$$

Note also that the parafermionic symmetries (2.8) imply that these models are in fact $\mathbb{Z}_{k}$ symmetric. 


\begin{tabular}{|c|c|c|c|c|c|c|c|c|c|c|}
\hline$(\ell, m)$ & $(0,0)$ & $(0,2)$ & $(0,4)$ & $(0,6)$ & $(2,0)$ & $(2,2)$ & $(1,1)$ & $(1,3)$ & $(1,5)$ & $(1,7)$ \\
\hline$(0,0)$ & $\chi_{(0,0)}$ & $\chi_{(0,2)}$ & $\chi_{(0,4)}$ & $\chi_{(0,6)}$ & $\chi_{(2,0)}$ & $\chi_{(2,2)}$ & $\chi_{(1,1)}$ & $\chi_{(1,3)}$ & $\chi_{(1,5)}$ & $\chi_{(1,7)}$ \\
\hline$(0,2)$ & $\chi_{(0,6)}$ & $\chi_{(0,0)}$ & $\chi_{(0,2)}$ & $\chi_{(0,4)}$ & $\chi_{(2,2)}$ & $\chi_{(2,0)}$ & $\chi_{(1,7)}$ & $\chi_{(1,1)}$ & $\chi_{(1,3)}$ & $\chi_{(1,5)}$ \\
\hline$(0,4)$ & $\chi_{(0,4)}$ & $\chi_{(0,6)}$ & $\chi_{(0,0)}$ & $\chi_{(0,2)}$ & $\chi_{(2,0)}$ & $\chi_{(2,2)}$ & $\chi_{(1,5)}$ & $\chi_{(1,7)}$ & $\chi_{(1,1)}$ & $\chi_{(1,3)}$ \\
\hline$(0,6)$ & $\chi_{(0,2)}$ & $\chi_{(0,4)}$ & $\chi_{(0,6)}$ & $\chi_{(0,0)}$ & $\chi_{(2,2)}$ & $\chi_{(2,0)}$ & $\chi_{(1,3)}$ & $\chi_{(1,5)}$ & $\chi_{(1,7)}$ & $\chi_{(1,1)}$ \\
\hline$(2,0)$ & $\chi_{(2,0)}$ & $\chi_{(2,2)}$ & $\chi_{(2,0)}$ & $\chi_{(2,2)}$ & $\chi_{(0,0)}+\chi_{(2,0)}+\chi_{(4,0)}$ & $\chi_{(0,2)}+\chi_{(2,2)}+\chi_{(4,2)}$ & $\chi_{(1,1)}+\chi_{(1,5)}$ & $\chi_{(1,3)}+\chi_{(1,7)}$ & $\chi_{(1,1)}+\chi_{(1,5)}$ & $\chi_{(1,3)}+\chi_{(1,7)}$ \\
\hline$(2,2)$ & $\chi_{(2,2)}$ & $\chi_{(2,0)}$ & $\chi_{(2,2)}$ & $\chi_{(2,0)}$ & $\chi_{(0,2)}+\chi_{(2,2)}+\chi_{(4,2)}$ & $\chi_{(0,0)}+\chi_{(2,0)}+\chi_{(4,0)}$ & $\chi_{(1,3)}+\chi_{(1,7)}$ & $\chi_{(1,1)}+\chi_{(1,5)}$ & $\chi_{(1,3)}+\chi_{(1,7)}$ & $\chi_{(1,1)}+\chi_{(1,5)}$ \\
\hline$(1,1)$ & $\chi_{(1,7)}$ & $\chi_{(1,1)}$ & $\chi_{(1,3)}$ & $\chi_{(1,5)}$ & $\chi_{(1,3)}+\chi_{(1,7)}$ & $\chi_{(1,1)}+\chi_{(1,5)}$ & $\chi_{(0,0)}+\chi_{(2,0)}$ & $\chi_{(0,2)}+\chi_{(2,2)}$ & $\chi_{(0,4)}+\chi_{(2,0)}$ & $\chi_{(0,6)}+\chi_{(2,2)}$ \\
\hline$(1,3)$ & $\chi_{(1,5)}$ & $\chi_{(1,7)}$ & $\chi_{(1,1)}$ & $\chi_{(1,3)}$ & $\chi_{(1,1)}+\chi_{(1,5)}$ & $\chi_{(1,3)}+\chi_{(1,7)}$ & $\chi_{(0,6)}+\chi_{(2,2)}$ & $\chi_{(0,0)}+\chi_{(2,0)}$ & $\chi_{(0,2)}+\chi_{(2,2)}$ & $\chi_{(0,4)}+\chi_{(2,0)}$ \\
\hline$(1,5)$ & $\chi_{(1,3)}$ & $\chi_{(1,5)}$ & $\chi_{(1,7)}$ & $\chi_{(1,1)}$ & $\chi_{(1,3)}+\chi_{(1,7)}$ & $\chi_{(1,1)}+\chi_{(1,5)}$ & $\chi_{(0,4)}+\chi_{(2,0)}$ & $\chi_{(0,6)}+\chi_{(2,2)}$ & $\chi_{(0,0)}+\chi_{(2,0)}$ & $\chi_{(0,2)}+\chi_{(2,2)}$ \\
\hline$(1,7)$ & $\chi_{(1,1)}$ & $\chi_{(1,3)}$ & $\chi_{(1,5)}$ & $\chi_{(1,7)}$ & $\chi_{(1,1)}+\chi_{(1,5)}$ & $\chi_{(1,3)}+\chi_{(1,7)}$ & $\chi_{(0,2)}+\chi_{(2,2)}$ & $\chi_{(0,4)}+\chi_{(2,0)}$ & $\chi_{(0,6)}+\chi_{(2,2)}$ & $\chi_{(0,0)}+\chi_{(2,0)}$ \\
\hline
\end{tabular}

Table 5: Cylinder partition functions $Z_{L \mid R}$ for $\left(A_{5}, \mathbb{Z}_{8}\right)$. The rows are labelled by the left boundary condition and the columns by the right boundary condition. The table is in fact symmetric due to conjugation symmetry.

\begin{tabular}{c|cccccc}
$(a, m)$ & $(A, 0)$ & $(A, 2)$ & $(B, 0)$ & $(B, 2)$ & $(O, 1)$ & $(O, 3)$ \\
\hline$(A, 0)$ & $\chi_{(0,0)}+\chi_{(4,0)}$ & $2 \chi_{(0,2)}$ & $\chi_{(2,0)}$ & $\chi_{(2,2)}$ & $\chi_{(1,1)}+\chi_{(1,3)}$ & $\chi_{(1,1)}+\chi_{(1,3)}$ \\
$(A, 2)$ & $2 \chi_{(0,2)}$ & $\chi_{(0,0)}+\chi_{(4,0)}$ & $\chi_{(2,2)}$ & $\chi_{(2,0)}$ & $\chi_{(1,1)}+\chi_{(1,3)}$ & $\chi_{(1,1)}+\chi_{(1,3)}$ \\
$(B, 0)$ & $\chi_{(2,0)}$ & $\chi_{(2,2)}$ & $\chi_{(0,0)}+\chi_{(4,0)}$ & $2 \chi_{(0,2)}$ & $\chi_{(1,1)}+\chi_{(1,3)}$ & $\chi_{(1,1)}+\chi_{(1,3)}$ \\
$(B, 2)$ & $\chi_{(2,2)}$ & $\chi_{(2,0)}$ & $2 \chi_{(0,2)}$ & $\chi_{(0,0)}+\chi_{(4,0)}$ & $\chi_{(1,1)}+\chi_{(1,3)}$ & $\chi_{(1,1)}+\chi_{(1,3)}$ \\
$(O, 1)$ & $\chi_{(1,1)}+\chi_{(1,3)}$ & $\chi_{(1,1)}+\chi_{(1,3)}$ & $\chi_{(1,1)}+\chi_{(1,3)}$ & $\chi_{(1,1)}+\chi_{(1,3)}$ & $\chi_{(0,0)}+2 \chi_{(2,0)}+\chi_{(4,0)}$ & $2 \chi_{(0,2)}+2 \chi_{(2,2)}$ \\
$(O, 3)$ & $\chi_{(1,1)}+\chi_{(1,3)}$ & $\chi_{(1,1)}+\chi_{(1,3)}$ & $\chi_{(1,1)}+\chi_{(1,3)}$ & $\chi_{(1,1)}+\chi_{(1,3)}$ & $2 \chi_{(0,2)}+2 \chi_{(2,2)}$ & $\chi_{(0,0)}+2 \chi_{(2,0)}+\chi_{(4,0)}$
\end{tabular}

Table 6: Cylinder partition functions $Z_{L \mid R}$ for $\left(D_{4}, \mathbb{Z}_{8}\right)$. $O$ stands for the center point of $D_{4}$ and $A, B$ for any two of the three other points. The table is $\mathbb{Z}_{3}$ symmetric. 


$$
\ell \in A_{5}
$$

$$
A_{5} \quad(k=4)
$$

$D_{4} \quad(k=4)$
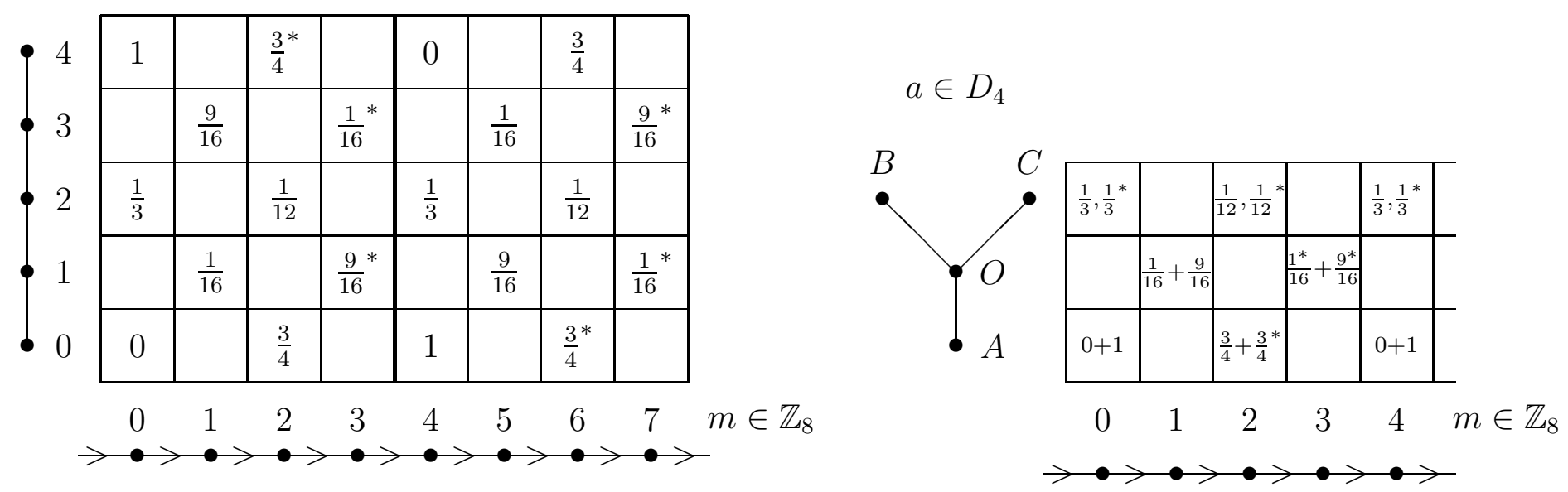

Table 7: Conformal grid for the $\left(D_{4}, \mathbb{Z}_{8}\right)$ model resulting from the folding of the $\left(A_{5}, \mathbb{Z}_{8}\right)$ model. $A, B, C$ are even, $O$ is odd.

$$
\begin{array}{ll}
\hat{\chi}_{(A, 0)}=\chi_{(0,0)}+\chi_{(4,0)} & \hat{\chi}_{(A, 2)}=\chi_{(0,2)}+\chi_{(4,2)} \\
\hat{\chi}_{(O, 1)}=\chi_{(1,1)}+\chi_{(1,5)} & \hat{\chi}_{(O, 3)}=\chi_{(1,7)}+\chi_{(1,3)} \\
\hat{\chi}_{(B, 0)}=\chi_{(2,0)} & \hat{\chi}_{(B, 2)}=\chi_{(2,2)} \\
\hat{\chi}_{(C, 0)}=\chi_{(2,0)} & \hat{\chi}_{(C, 2)}=\chi_{(2,2)}
\end{array}
$$

\begin{tabular}{c|cccccccc}
$(a, m)$ & $(A, 0)$ & $(A, 2)$ & $(B, 0)$ & $(B, 2)$ & $(C, 0)$ & $(C, 2)$ & $(O, 1)$ & $(O, 3)$ \\
\hline$(A, 0)$ & $\hat{\chi}_{(A, 0)}$ & $\hat{\chi}_{(A, 2)}$ & $\hat{\chi}_{(B, 0)}$ & $\hat{\chi}_{(B, 2)}$ & $\hat{\chi}_{(C, 0)}$ & $\hat{\chi}_{(C, 2)}$ & $\hat{\chi}_{(O, 1)}$ & $\hat{\chi}_{(O, 3)}$ \\
$(A, 2)$ & $\hat{\chi}_{(A, 2)}$ & $\hat{\chi}_{(A, 0)}$ & $\hat{\chi}_{(B, 2)}$ & $\hat{\chi}_{(B, 0)}$ & $\hat{\chi}_{(C, 2)}$ & $\hat{\chi}_{(C, 0)}$ & $\hat{\chi}_{(O, 3)}$ & $\hat{\chi}_{(O, 1)}$ \\
$(B, 0)$ & $\hat{\chi}_{(C, 0)}$ & $\hat{\chi}_{(C, 2)}$ & $\hat{\chi}_{(A, 0)}$ & $\hat{\chi}_{(A, 2)}$ & $\hat{\chi}_{(B, 0)}$ & $\hat{\chi}_{(B, 2)}$ & $\hat{\chi}_{(O, 1)}$ & $\hat{\chi}_{(O, 3)}$ \\
$(B, 2)$ & $\hat{\chi}_{(C, 2)}$ & $\hat{\chi}_{(C, 0)}$ & $\hat{\chi}_{(A, 2)}$ & $\hat{\chi}_{(A, 0)}$ & $\hat{\chi}_{(B, 2)}$ & $\hat{\chi}_{(B, 0)}$ & $\hat{\chi}_{(O, 3)}$ & $\hat{\chi}_{(O, 1)}$ \\
$(C, 0)$ & $\hat{\chi}_{(B, 0)}$ & $\hat{\chi}_{(B, 2)}$ & $\hat{\chi}_{(C, 0)}$ & $\hat{\chi}_{(C, 2)}$ & $\hat{\chi}_{(A, 0)}$ & $\hat{\chi}_{(A, 2)}$ & $\hat{\chi}_{(O, 1)}$ & $\hat{\chi}_{(O, 3)}$ \\
$(C, 2)$ & $\hat{\chi}_{(B, 2)}$ & $\hat{\chi}_{(B, 0)}$ & $\hat{\chi}_{(C, 2)}$ & $\hat{\chi}_{(C, 0)}$ & $\hat{\chi}_{(A, 2)}$ & $\hat{\chi}_{(A, 0)}$ & $\hat{\chi}_{(O, 3)}$ & $\hat{\chi}_{(O, 1)}$ \\
$(O, 1)$ & $\hat{\chi}_{(O, 3)}$ & $\hat{\chi}_{(O, 1)}$ & $\hat{\chi}_{(O, 3)}$ & $\hat{\chi}_{(O, 1)}$ & $\hat{\chi}_{(O, 3)}$ & $\hat{\chi}_{(O, 1)}$ & $\hat{\chi}_{(A, 0)}+\hat{\chi}_{(B, 0)}+\hat{\chi}_{(C, 0)}$ & $\hat{\chi}_{(A, 2)}+\hat{\chi}_{(B, 2)}+\hat{\chi}_{(C, 2)}$ \\
$(O, 3)$ & $\hat{\chi}_{(O, 1)}$ & $\hat{\chi}_{(O, 3)}$ & $\hat{\chi}_{(O, 1)}$ & $\hat{\chi}_{(O, 3)}$ & $\hat{\chi}_{(O, 1)}$ & $\hat{\chi}_{(O, 3)}$ & $\hat{\chi}_{(A, 2)}+\hat{\chi}_{(B, 2)}+\hat{\chi}_{(C, 2)}$ & $\hat{\chi}_{(A, 0)}+\hat{\chi}_{(B, 0)}+\hat{\chi}_{(C, 0)}$
\end{tabular}

Table 8: Cylinder partition functions (fusion algebra) for $\left(D_{4}, \mathbb{Z}_{8}\right)$ in terms of extended characters. $A$ is the distinguished node. 
The set of boundary conditions $(a, m)$ for $D$ and $E$ theories gives rise to an extended fusion algebra of conformal fields $\hat{\varphi}_{(a, m)}$, namely the graph fusion algebra

$$
\hat{\varphi}_{(a, m)} \times \hat{\varphi}_{\left(a^{\prime}, m^{\prime}\right)}=\sum_{\left(a^{\prime \prime}, m^{\prime \prime}\right)} \hat{N}_{(a, m)\left(a^{\prime}, m^{\prime}\right)}{ }^{\left(a^{\prime \prime}, m^{\prime \prime}\right)} \hat{\varphi}_{\left(a^{\prime \prime}, m^{\prime \prime}\right)}
$$

with structure constants given by the Verlinde like formula

$$
\hat{N}_{\left(a_{1}, m_{1}\right)\left(a_{2}, m_{2}\right)}{ }^{\left(a_{3}, m_{3}\right)}=\sum_{\left(a^{\prime}, m\right)} \frac{\Psi_{\left(a_{1}, m_{1}\right)}{ }^{\left(a^{\prime}, m\right)} \Psi_{\left(a_{2}, m_{2}\right)}^{\left(a^{\prime}, m\right)} \Psi_{\left(a_{3}, m_{3}\right)}\left(a^{\prime}, m\right)^{*}}{\Psi_{(1,0)}\left(a^{\prime}, m\right)}
$$

where the sum on $\left(a^{\prime}, m\right) \in \operatorname{Exp}(G) \times \mathbb{Z}_{2 k}$ is over the fundamental domain. In terms of the extended characters, the cylinder partition functions can be written as

$$
Z_{(a, m) \mid\left(a^{\prime}, m^{\prime}\right)}(q)=\sum_{\left(a^{\prime \prime}, m^{\prime \prime}\right)} \hat{N}_{(a, m)^{*}\left(a^{\prime}, m^{\prime}\right)}^{\left(a^{\prime \prime}, m^{\prime \prime}\right)} \hat{\chi}_{\left(a^{\prime \prime}, m^{\prime \prime}\right)}(q)
$$

Table 8 gives the example of the $D_{4}$ case. In this form the partition functions are no longer

explicitly $\mathbb{Z}_{3}$ symmetric. There is a distinguished node which corresponds to the identity operator of the fusion algebra which for $D_{4}$ is $(A, 0)$.

\section{Lattice Realizations of $\mathbb{Z}_{k}$ Parafermions}

\section{1 $A-D-E$ lattice models and integrable boundaries}

The principal $\mathbb{Z}_{k}$ theories are realized as the continuum scaling limit of the critical $A-D-E$ lattice models [9] with negative spectral parameter. In these models the spin states are taken to be the nodes of the graph $G$ of $A, D$ or $E$ type. Let $g$ be the Coxeter number of $G$. Then the bulk face weights are

$$
W\left(\begin{array}{ll|l}
d & c & u \\
a & b & u
\end{array}\right)={ }_{a}^{d}{ }_{b}^{c}=\frac{\sin (\lambda-u)}{\sin \lambda} \delta_{a c}+\frac{\sin u}{\sin \lambda} \frac{\sqrt{\psi_{a} \psi_{c}}}{\psi_{b}} \delta_{b d},
$$

where $u$ is the spectral parameter, $\lambda=\pi / g$ is the crossing parameter and the weights are understood to vanish if the adjacency condition of $A, D$ or $E$ type is not satisfied along the four edges of the face. The crossing factors $\psi_{a}$ are the entries of the Perron-Frobenius eigenvector of the adjacency matrix $G$. If $0<u<\lambda$ the continuum scaling limit of these models describes the $s \ell(2)$ unitary minimal models. Otherwise, if $\lambda-\pi / 2<u<0$, the continuum scaling limit describes the principal $\mathbb{Z}_{k}$ parafermions with $k=g-2$.

The $A-D$ - $E$ models are also integrable in the presence of a boundary [22]. The integrable boundary conditions at a conformal point are labelled [4] by $(r, a) \in\left(A_{g-2}, G\right)$. A general expression for the boundary weights of the $(r, a)$ boundary condition [4] in terms of boundary 
edge weights is

$$
\begin{aligned}
& B^{r a}\left(\begin{array}{cc}
c & \delta \\
b & \beta
\end{array} \mid u, \xi\right)=\left.c \underbrace{r, a}_{b} u\right|_{\beta} ^{d} \\
& =\frac{\sin (\xi-u) \sin (\xi+u+r \lambda) \psi_{c}^{1 / 2}}{\sin (2 \xi) \psi_{b}^{1 / 2}} \delta_{b d} \delta_{\beta \delta}+\frac{\sin (2 u)}{\sin (2 \xi)} \sum_{\gamma=1}^{F_{c a}^{r+1}} E^{r a}(b, c)_{\beta \gamma} E^{r a}(d, c)_{\delta \gamma} .
\end{aligned}
$$

Here $\xi$ is a free parameter that should be thought of as a boundary field and $E^{r a}(b, c)_{\beta \gamma}$ are edge weights specified in Behrend and Pearce [4]. The fused adjacency matrices $F^{r}$ at level $r$ which appear in the summation are simply another notation for the intertwiners $F^{r}=V_{r}^{G}$ defined previously. These are given recursively in terms of the adjacency matrix $G$ of the $A-D-E$ graph by the $s \ell(2)$ fusion rules

$$
F^{1}=I, \quad F^{2}=G, \quad F^{r}=G F^{r-1}-F^{r-2}, \quad r=3, \ldots, g
$$

The labels $\alpha, \beta, \gamma$ are bond variables or degeneracies with $\beta=1, \ldots, F_{a b}^{r}, \delta=1, \ldots, F_{a d}^{r}$. At $u=\xi$ the boundary weights are independent of $\xi$ and decompose simply in terms of boundary edge weights

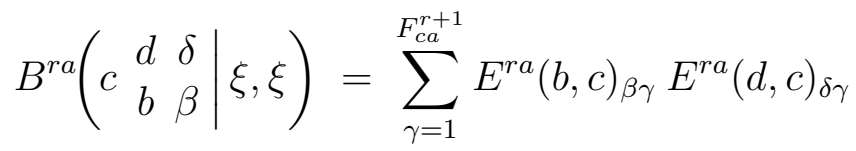

It is precisely at $u=\xi$, when the triangle boundary weights reduce effectively to edge weights, that the integrable boundary conditions correspond [4] to conformal boundary conditions.

The $A-D-E$ face weights and boundary weights satisfy the Yang-Baxter and boundary Yang-Baxter equations. This ensures integrability through commuting double row transfer matrices. The entries of the $N$ column double-row transfer matrix with boundary conditions $\left(r_{1}, a_{1}\right)$ on the left and $\left(r_{2}, a_{2}\right)$ on the right are given by

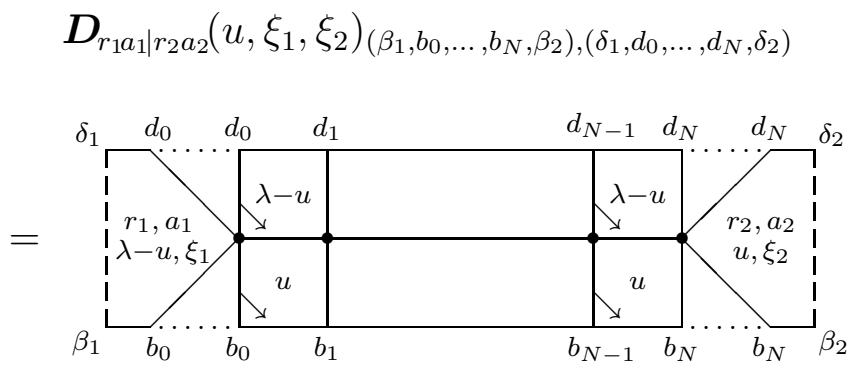

\subsection{Finite-size corrections}

The properties of the $A-D-E$ lattice models connect to the data of the associated conformal field theories through the finite-size corrections to the eigenvalues of the double row transfer matrices. If we write the eigenvalues of $\boldsymbol{D}_{r_{1} a_{1} \mid r_{2} a_{2}}\left(u, \xi_{1}, \xi_{2}\right)$ as

$$
D_{n}(u)=\exp \left(-E_{n}(u)\right), \quad n=0,1,2, \ldots
$$


then the finite-size corrections to the energies $E_{n}$ take the form

$$
E_{n}(u)=2 N f(u)+f_{r_{1}, a_{1} \mid r_{2}, a_{2}}(u)+\frac{2 \pi \sin \vartheta}{N}\left(-\frac{c}{24}+\Delta_{n}+k_{n}\right)+o\left(\frac{1}{N}\right), \quad k_{n} \in \mathbb{N}
$$

where $f(u)$ is the bulk free energy, $f_{r_{1}, a_{1} \mid r_{2}, a_{2}}(u)$ is the boundary free energy, $c$ is the central charge, $\Delta_{n}$ is a conformal weight and the anisotropy angle is given by

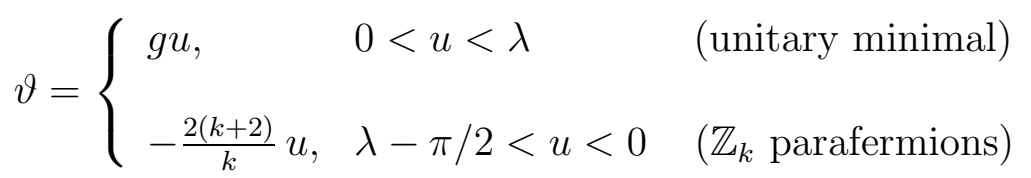

where $g=k+2$ is the Coxeter number.

Removing the bulk and boundary contributions to the partition function on a cylinder leads to the conformal partition function $Z_{j \mid k}(q)$ with left and right boundaries $j=\left(r_{1}, a_{1}\right)$, $k=\left(r_{2}, a_{2}\right)$. This can be expressed as a linear form in characters

$$
Z_{j \mid k}(q)=\sum_{i} n_{i j}{ }^{k} \chi_{i}(q)
$$

where $i$ is summed over the primary fields and the integers $n_{i j}{ }^{k} \in \mathbb{N}$ give the operator content. For $M$ double rows the modular parameter is

$$
q=\exp (2 \pi i \tau), \quad \tau=i \frac{M}{N} \sin \vartheta
$$

where $M / N$ is the aspect ratio of the cylinder.

For a system to be conformally invariant it is usually demanded that it is both isotropic and translationally invariant. For the unitary minimal $A-D-E$ models with $0<u<\lambda$ the geometry is that of an isotropic square lattice when $u=\lambda / 2$ and $\vartheta=\pi / 2$. In this case the alternation of rows in the double row transfer matrix also disappears since $\lambda-u=u$. So the conformal point occurs for

$$
\lambda-u=u=\xi_{1}=\xi_{2}=\lambda / 2 \quad \text { (unitary minimal models) }
$$

In contrast, for the $\mathbb{Z}_{k}$ parafermionic $A-D$ - $E$ models with $\lambda-\pi / 2<u<0$, the relevant geometry is not that of an isotropic square lattice. Instead, for $\mathbb{Z}_{k}$ parafermions, the relevant choice is $u=-\lambda$ with $\vartheta=2 \pi / k$. Thus for the $W_{3}$ or hard hexagon model the geometry is that of the triangular lattice with $\vartheta=2 \pi / 3$. In general it follows that $\lambda-u=2 \lambda$ so the alternation of rows in the double row transfer matrix persists even though the second row is still the transpose of the first row. Moreover, to ensure that the left and right boundary conditions are conformal we need to choose $\xi_{1}=2 \lambda$ and $\xi_{2}=-\lambda$ so the conformal point is

$$
\lambda-u=\xi_{1}=2 \lambda, \quad u=\xi_{2}=-\lambda \quad\left(\mathbb{Z}_{k} \text { parafermions }\right)
$$

Notice that at this conformal point the double row transfer matrix is not left-right symmetric and the boundary free energies for a given boundary condition $(r, a)$ on the left and right are different because they have different boundary fields $\xi_{1}=2 \lambda$ and $\xi_{2}=-\lambda$. 


\subsection{Bulk free energies}

In this section we obtain the bulk free energies $f(u)$ or equivalently the partition functions per site $\kappa(u)=\exp (-f(u))$. Two $A-D-E$ models sharing the same Coxeter number are related by intertwiners so their bulk free energies are the same. Their boundary free energies are also related. Thus we only need to find the free energies for the $A_{L}$ models.

For general $L=k+1$, the partition function per site of the $A_{L}$ model satisfies the inversion relation

$$
\kappa(u) \kappa(u+\lambda)=\frac{\sin (u+\lambda) \sin (u-\lambda)}{\sin ^{2} \lambda} \frac{\sin \frac{L+1}{L-1} u}{\sin \frac{L+1}{L-1}(u+\lambda)}:=q_{b}(u)
$$

and the crossing symmetry

$$
\kappa(u)=\kappa(\lambda-u)
$$

The inversion relation is established by keeping just the dominant terms in the TBA and inversion identity hierarchies [22], using height reversal symmetry and equating just the bulk terms of order $2 N$. The inversion relation, crossing symmetry and height reversal symmetry determine a unique solution which is analytic and non-zero in the analyticity

strip $\operatorname{Re}(u) \in\left(\frac{-\pi+\lambda}{2}, \frac{\lambda}{2}\right)$, which contains the physical strip $\operatorname{Re}(u) \in\left(-\frac{\pi}{2}+\lambda, 0\right)$ for the regime we consider. This problem has been solved, in a different context, by Baxter [24]. The solution for general $L$ can be written as an integral

$$
\kappa(u)=\exp \int_{-\infty}^{+\infty} \sinh u t \frac{\sinh (\pi-\lambda) t \sinh (\pi-3 \lambda+u) t-\sinh \lambda t \sinh (u+\lambda) t}{t \sinh \pi t \sinh (\pi-2 \lambda) t} d t .
$$

The solution can also be written explicitly for $L$ even ( $k$ odd) as

$$
\kappa(u)=\frac{\sin (u-2 \lambda)}{\sin \lambda} \prod_{k=1}^{L / 2-1} \frac{\sin (u+(2 k-1) \lambda)}{\sin (u+2 k \lambda)} \frac{\sin \frac{L+1}{L-1}(u+2 k \lambda)}{\sin \frac{L+1}{L-1}(u+(2 k-1) \lambda)} .
$$

\subsection{Boundary free energies}

In this section we obtain results for the boundary free energies by extending the inversion relation method [25, 26]. The boundary free energy contribution comes in three different parts

$$
f_{r, s \mid r^{\prime}, s^{\prime}}=f_{0}+f_{r, s}^{L}+f_{r^{\prime}, s^{\prime}}^{R}
$$

The vacuum term $f_{0}$ does not depend on the boundary condition. It is free of zeros and its logarithm is analytic on the physical analyticity strip. The remaining contributions $f_{r, s}^{L / R}$ for each boundary condition $(r, s)$ are different on the left $L$ and on the right $R$ and exhibit zeros in the physical analyticity strip. The boundary free energies are obtained by repeating the analysis of the TBA and inversion identity hierarchies [22 keeping the dominant terms as 
in the bulk calculation but this time equating the boundary terms of order 1 in the physical analyticity strip $\operatorname{Re}(u) \in\left(\frac{-\pi+\lambda}{2},-\frac{\lambda}{2}\right)$.

Let $q_{b}$ be the RHS of the bulk inversion relation (3.13). Then the vacuum functional equation we obtain is, with $\kappa_{0}(u)=\exp \left(-f_{0}(u)\right)$,

$$
\kappa_{0}(u) \kappa_{0}(u+\lambda)=q_{b}(u) q_{b}\left(-\frac{\pi}{2}-u\right) \frac{4 \sin ^{4} \lambda}{\sin (\lambda-2 u) \sin (\lambda-2(u+\lambda))} .
$$

Hence we find the solution with the required analyticity is expressed in terms of the bulk free energy as

$$
\kappa_{0}(u)=\kappa(u) \kappa\left(-\frac{\pi}{2}-u+\lambda\right) \frac{2 \sin ^{2} \lambda}{\sin (\lambda-2 u)}
$$

For $L$ even, one has an explicit expression

$$
\begin{aligned}
\kappa_{0}(u)= & \frac{\sin 2(u+\lambda)}{\sin (\lambda-2 u)} \\
& \times \prod_{k=1}^{\frac{L}{2}-1} \frac{\sin 2(u+(2 k+1) \lambda)}{\sin 2(u+2 k \lambda)} \frac{\sin \frac{L+1}{L-1}(u+2 k \lambda)}{\sin \frac{L+1}{L-1}(u+(2 k-1) \lambda)} \frac{\cos \frac{L+1}{L-1}(u+(2 k-1) \lambda)}{\cos \frac{L+1}{L-1}(u+2 k \lambda)}
\end{aligned}
$$

In this case the inversion relation admits other explicit solutions. Combining the zeros and poles of the two bulk free energy terms for $L \equiv 1 \bmod 4$, we find

$$
\begin{aligned}
\kappa_{0}(u)= & \frac{\sin 2\left(u+\left(\frac{L-1}{2}-1\right) \lambda\right)}{\sin (2 u-\lambda)} \frac{\sin \frac{L+1}{L-1}(u-\lambda)}{\sin \frac{L+1}{L-1}\left(u+\left(\frac{L-1}{2}-1\right) \lambda\right)} \\
& \times \prod_{k=1}^{\frac{L-1}{4}-1} \frac{\sin 2(u+(2 k-1) \lambda)}{\sin 2(u+2 k \lambda)} \frac{\sin \frac{L+1}{L-1}(u+2 k \lambda)}{\sin \frac{L+1}{L-1}(u+(2 k-1) \lambda)} \frac{\cos \frac{L+1}{L-1}(u+(2 k-1) \lambda)}{\cos \frac{L+1}{L-1}(u+2 k \lambda)}
\end{aligned}
$$

In the remaining case $L \equiv 3 \bmod 4$, the vacuum boundary free energy can only be expressed in terms of integrals. Let $q_{0}$ be the RHS of the functional equation (3.18). It is Analytic and Non-Zero in the strip $\operatorname{Re}(u) \in\left(\frac{-\pi+\lambda}{2},-\frac{\lambda}{2}\right)$. Furthermore the derivative $f_{0}^{\prime}$ approaches a Constant when $\operatorname{Im}(u) \rightarrow \pm \infty$ (ANZC). Hence we can introduce the Fourier transforms of the derivatives

$$
\begin{aligned}
\mathcal{F}_{0}(k) & :=\underset{\substack{-\pi+\lambda \\
\frac{-\pi+\lambda}{2}<\operatorname{Re}(u)<-\frac{\lambda}{2}}}{ } f_{0}^{\prime}(u) e^{-k u} d u \\
\frac{d}{d u} f_{0}(u) & =\int_{-\infty}^{+\infty} \mathcal{F}_{0}(k) e^{k u} d k
\end{aligned}
$$

so that 3.18 becomes

$$
\mathcal{F}_{0}(k)\left(1+e^{k \lambda}\right)=\frac{1}{2 i \pi} \int \frac{q_{0}^{\prime}(u)}{q_{0}(u)} e^{-k u} d u
$$


The solution by inverse Fourier transform gives

$$
\begin{aligned}
\frac{d}{d u} f_{0}(u) & =\frac{1}{2 i \pi} \int d w\left(\frac{d}{d u} \log q_{0}(u-w)\right) \frac{L+1}{\sin (L+1) w} \\
0<\operatorname{Re}(w)<\min \left(\lambda, u+\frac{L}{2} \lambda\right) &
\end{aligned}
$$

Integrating with respect to $u$ and taking the $w$ integration along the vertical line $\operatorname{Re}(w)=$ $\epsilon>0$ we obtain in the limit $\epsilon \rightarrow 0$

$$
f_{0}(u)=\frac{\log q_{0}(u)}{2}+\frac{L+1}{\pi} \int_{0}^{\infty} \frac{\operatorname{Im} \log q_{0}(u-i w)}{\sinh (L+1) w} d w
$$

The boundary free energy $f_{(r, s)}$ arises from a boundary condition of type $(r, s)$ with a boundary field $\xi$. It does not depend on $s$. An integral form analogous to (3.26) can be derived with $q_{0}$ being replaced by the RHS of the boundary inversion relation

$$
\begin{aligned}
\kappa_{(r, s)}(u) \kappa_{(r, s)}(u+\lambda)= & \frac{\sin (u+\xi) \sin (u-\xi) \sin (u+\xi+r \lambda) \sin (u-\xi-r \lambda)}{\sin ^{4} \lambda} \\
& \times \frac{\sin \frac{L+1}{L-1}(u-\xi-(r-1) \lambda)}{\sin \frac{L+1}{L-1}(u-\xi)} \frac{\sin \frac{L+1}{L-1}(u+\xi+\lambda)}{\sin \frac{L+1}{L-1}(u+\xi+r \lambda)}
\end{aligned}
$$

The solution must be analytic on the analyticity strip with the same zeros as

$$
\sin (u+\xi) \sin (u-\xi-r \lambda)
$$

For $r$ odd, this inversion relation admits the explicit solution

$$
\begin{aligned}
\kappa_{(r, s)}(u)= & \frac{\sin (u+\xi) \sin (u-\xi-r \lambda)}{\sin ^{2} \lambda} \\
& \times \prod_{k=1}^{\frac{r-1}{2}} \frac{\sin (u+\xi+2 k \lambda)}{\sin (u+\xi+(2 k-1) \lambda)} \frac{\sin (u-\xi-(2 k-1) \lambda)}{\sin (u-\xi-2 k \lambda)} \\
& \quad \times \frac{\sin \frac{L+1}{L-1}(u+\xi+(2 k-1) \lambda)}{\sin \frac{L+1}{L-1}(u+\xi+2 k \lambda)} \frac{\sin \frac{L+1}{L-1}(u-\xi-2 k \lambda)}{\sin \frac{L+1}{L-1}(u-\xi-(2 k-1) \lambda)}
\end{aligned}
$$

with zeros at $u=-\xi, \xi+r \lambda,-\xi-\pi+\lambda, \xi-\pi+(r+1) \lambda$. Since these last two zeros are not desired, the formula is only valid when these points are outside the analyticity strip, i.e.,

$$
\frac{-\pi+\lambda}{2}<\operatorname{Re}(\xi)<\frac{\pi-\lambda}{2}-r \lambda .
$$

There is another solution when $L+r$ is even

$$
\begin{aligned}
\kappa_{(r, s)}(u)= & \frac{\sin (u+\xi-\lambda) \sin (u-\xi)}{\sin ^{2} \lambda} \\
& \times \prod_{k=1}^{\frac{L-r}{2}} \frac{\sin (u+\xi+(2(k-1)+r) \lambda)}{\sin (u+\xi+(2 k-1+r) \lambda)} \frac{\sin (u-\xi+2 k \lambda)}{\sin (u-\xi+(2 k-1) \lambda)} \\
& \quad \times \frac{\sin \frac{L+1}{L-1}(u+\xi+(2 k-1+r) \lambda)}{\sin \frac{L+1}{L-1}(u+\xi+(2(k-1)+r) \lambda)} \frac{\sin \frac{L+1}{L-1}(u-\xi+(2 k-1) \lambda)}{\sin \frac{L+1}{L-1}(u-\xi+2(k-1) \lambda)}
\end{aligned}
$$


with zeros at $u=-\xi, \xi+r \lambda, \xi+(r-1) \lambda-\pi,-\xi-(r-2) \lambda-\pi$. Clearly, this formula is only valid when

$$
\frac{-\pi+3 \lambda}{2}-r \lambda<\operatorname{Re}(\xi)<\frac{\pi-\lambda}{2}-r \lambda
$$

In the $A_{4}$ and $A_{5}$ cases, these formulas allow us to compute the boundary free energies. The values for $A_{5}$ are listed in Table 9 .

$$
\begin{array}{rlccc}
f_{0}(-\lambda / 2)= & \log \frac{1+\sqrt{2}}{3} & \\
r= & 1 & 2 & 3 & 4 \\
f_{(r, s)}(-\lambda / 2)= & 0 & \frac{1}{2} \log \frac{8}{3} & \frac{1}{2} \log 3 & \frac{1}{2} \log \frac{3}{2} \\
f_{(r, s)}(3 \lambda / 2)= & 0 & \frac{1}{2} \log 2 & \log \frac{4}{3} & \frac{1}{2} \log \frac{2}{3}
\end{array}
$$

Table 9: The right and left boundary free energies $f_{(r, s)}^{R}=f_{(r, s)}(u)$ and $f_{(r, s)}^{L}=f_{(r, s)}(\lambda-u)$ of the $A_{5}$ model at the special point $u=-\lambda / 2$ and $\lambda-u=3 \lambda / 2$. The boundary free energies are independent of $s$.

Some symmetries of the parafermion models are reflected in the boundary free energies. The left/right symmetry is broken in general but the usual height reversal symmetry $r, s \mapsto$ $r, L+1-s$ remains. Another symmetry comes from the fact that at a conformal point the boundary weights factorize into edge weights. The interchange of the two independent sublattices is then implemented by the Kac table symmetry $r, s \mapsto L-r, L+1-s$.

\section{Identifying integrable and conformal boundary con- ditions}

For the unitary minimal $A$ - $D$ - $E$ models with $0<u<\lambda$ there is a one-to-one correspondence [4] between integrable and conformal boundary conditions labelled by $(r, a) \in$ $\left(A_{g-2}, G\right)$ that respect the symmetry of the generalized Kac table. In contrast, for the $\mathbb{Z}_{k}$ parafermionic $A-D-E$ models with $\lambda-\pi / 2<u<0$, the situation is more subtle and the conformal labels $(a, m) \in\left(G, \mathbb{Z}_{2 k}\right)$ do not coincide with the natural labels $(r, a)$ arising from the construction [4] of integrable boundary conditions at the conformal point. It is therefore necessary to make an identification of the integrable and conformal boundary conditions from numerical data.

For the $A$ type $\mathbb{Z}_{k}$ models the dominant or vacuum configuration is given by the $2 k$ periodic sawtooth configuration

$$
\boldsymbol{a}=\{\ldots, 1,2, \ldots, k, k+1, k, \ldots, 2,1, \ldots\}
$$


It is thus clear that the vacuum $(\ell, m)=(0,0)$ on the left and the right should be identified with the integrable boundary condition $(r, a)=(1,1)$ on the left and the right with the number of faces $N=0 \bmod 2 k$ and this is confirmed by numerical computation. Next, if we fix the boundary condition to the vacuum $(\ell, m)=(0,0)$ on the left or right we find from (2.12) that the cylinder partition function reduces to a single character

$$
Z_{(0,0) \mid(\ell, m)}(q)=Z_{(\ell, m) \mid(0,0)}(q)=\chi_{(\ell, m)}(q)
$$

If we fix $N \bmod 2 k$, this allows us to identify, up to possible ambiguities of conjugation, the integrable boundary condition $(r, s)$ on the left or right with the conformal labels $(\ell, m)$ from the numerically determined cylinder partition functions. Once these identifications are made they can be checked for consistency against all the other cylinder partition functions given in (2.12).

Proceeding in this way we find the following correspondence for the $A_{L}$ parafermions with $N=0 \bmod 2 k$

$$
(\ell, m)= \begin{cases}(s-1, r-1), & r+s \text { even } \\ (k+1-s, k-r), & r+s \text { odd }\end{cases}
$$

This is consistent with the Kac table symmetries, $(r, s) \mapsto(k+1-r, k+2-s)$ and (2.8), of both the minimal models and parafermions. The correspondence for the $A_{3}, A_{4}$ and $A_{5}$ parafermion models is listed explicitly in Table 10. The height reversal symmetry $s \mapsto$ $k+2-s$, flips the tables about their middle rows and corresponds at the level of the conformal algebra to a fusion with the field $\varphi_{(0, k)}$ which is of order two. The conjugacy $(l, m) \mapsto(k-l, k-m)$ can be seen in these tables as a central symmetry which centre has been indicated for the even sublattice. The centre of the usual Kac table symmetry of the minimal models has been indicated too.

For a number of faces $N \neq 0 \bmod 2 k$, the left/right symmetry of this correspondence is broken. Keeping the same correspondence between integrable boundary conditions $(r, s)$ and fields $(\ell, m)$ on the left hand side, the correspondence on the right hand side for a number of faces $N=1 \bmod 2 k$ is simply given by the same table where the columns are shifted cyclically to the left, the first column becoming the last column after a top/bottom flip as shown in Table 11] and so on for other mod properties. Therefore, for a number of faces $N=k \bmod 2 k$, the correspondence table is completely flipped about the middle row and we get the height reversed correspondence. This result is supported by our numerical computations for $k=2,3,4$ summarised in Section 5 .

This correspondence can be carried over to the $D$ and $E$ cases. For $N=0 \bmod k$, the result is again given by the trivial identification of $A_{k}$ with $\mathbb{Z}_{k}$ for the even sublattice and its flip for the odd sublattice. The cylinder partition functions in this case are obtained from the $A$ cases by intertwiners [4]

$$
Z_{r_{1} a_{1} \mid r_{2} a_{2}}(q)=\sum_{s=1}^{L} V_{s a_{1}}{ }^{a_{2}} Z_{r_{1} 1 \mid r_{2} s}^{A_{L}}(q)
$$


$A_{3} \quad(k=2)$

$s \in A_{3}$

$\ell \in A_{3}$

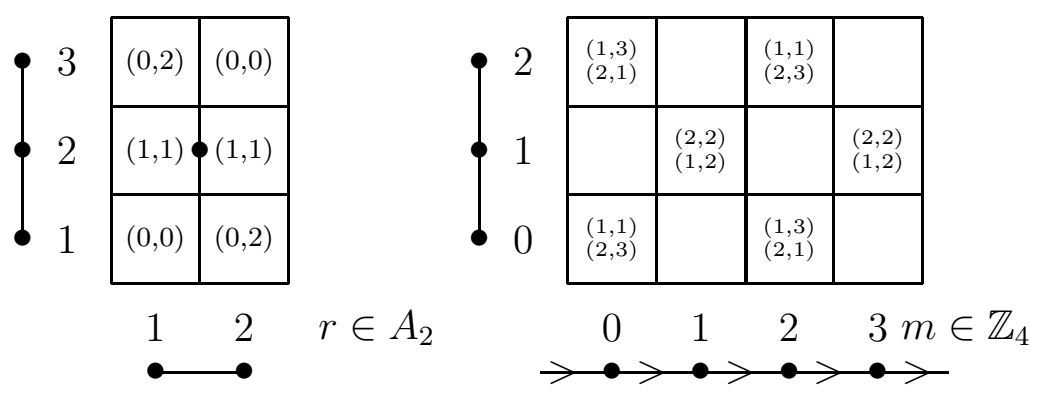

$A_{4} \quad(k=3)$
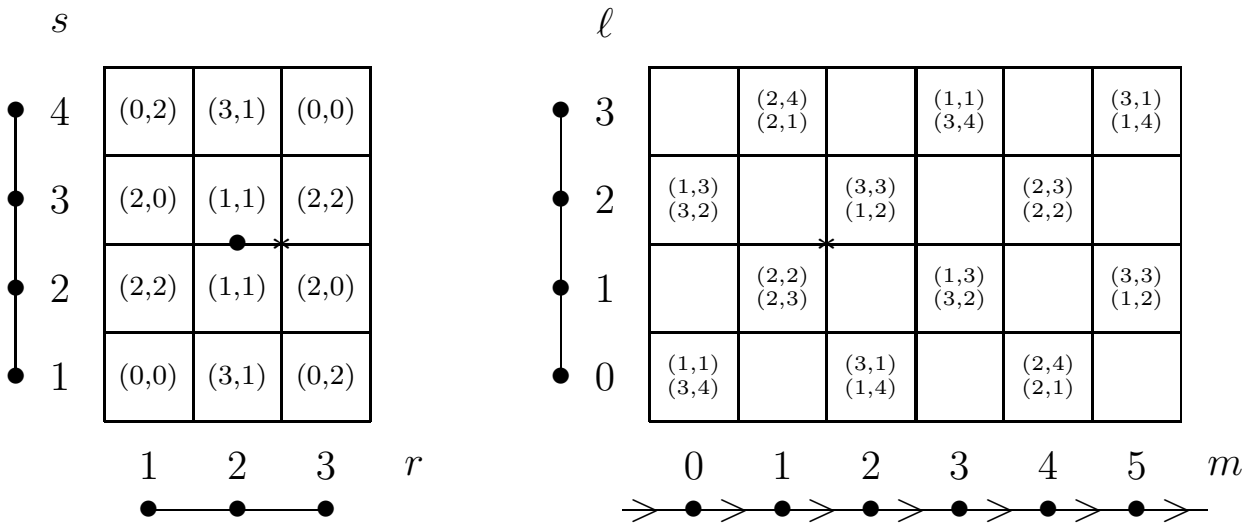

$S$

$A_{5} \quad(k=4)$

\begin{tabular}{|l|l|l|l|l|}
5 & $(4,0)$ & $(0,2)$ & $(4,2)$ & $(0,0)$ \\
\cline { 2 - 5 } & $(1,3)$ & $(3,1)$ & $(1,1)$ & $(3,3)$ \\
\hline 3 & $(2,0)$ & $(2,2)$ & $(2 * 2)$ & $(2,0)$ \\
\hline 2 & $(3,3)$ & $(1,1)$ & $(3,1)$ & $(1,3)$ \\
\hline 1 & $(0,0)$ & $(4,2)$ & $(0,2)$ & $(4,0)$ \\
\hline
\end{tabular}

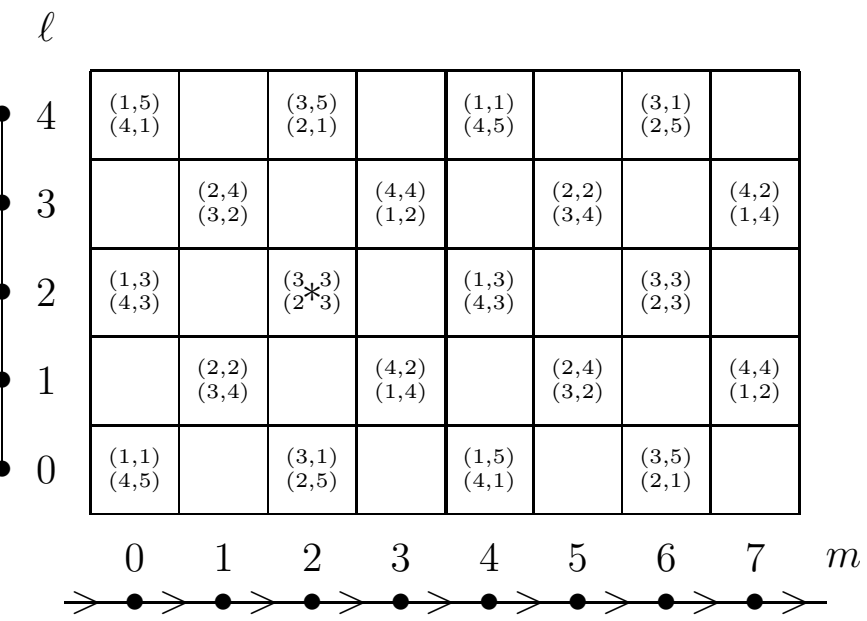

Table 10: The correspondence $(r, s) \mapsto(\ell, m)$ between the construction labels $(r, s) \in A_{k} \times$ $A_{k+1}$ of the integrable boundary conditions and the parafermionic conformal fields $(\ell, m) \in$ $A_{k+1} \times \mathbb{Z}_{2 k}$ and its inverse, for $k=2,3,4$ and $N=0 \bmod 2 k$. The inverse is a 1:2 mapping. The conjugation is realized in the even sub-lattices by a central inversion symmetry through the point indicated by $*$, and the minimal model symmetry centre is indicated by $\bullet$. 


\begin{tabular}{|c|c|c|c|c|c|c|c|c|c|c|c|c|c|}
\hline \multicolumn{4}{|c|}{${ }_{s} A_{3} \quad(k=2)$} & \multicolumn{5}{|c|}{$A_{4} \quad(k=3)$} & & \multicolumn{4}{|c|}{$A_{5} \quad(k=4)$} \\
\hline 3 & $(0,0)$ & $(0,0)$ & & 4 & $(3,1)$ & $(0,0)$ & $(0,0)$ & & 5 & $(0,2)$ & $(4,2)$ & $(0,0)$ & $(0,0)$ \\
\hline 2 & $(1,1)$ & $(1,1)$ & & 3 & $(1,1)$ & $(2,2)$ & $(2,2)$ & & 4 & $(3,1)$ & $(1,1)$ & $(3,3)$ & $(3,3)$ \\
\hline 1 & $(0,2)$ & $(0,2)$ & & 2 & $(1,1)$ & $(2,0)$ & $(2,0)$ & & 3 & $(2,2)$ & $(2,2)$ & $(2,0)$ & $(2,0)$ \\
\hline & 1 & 2 & $r$ & 1 & $(3,1)$ & $(0,2)$ & $(0,2)$ & & 2 & $(1,1)$ & $(3,1)$ & $(1,3)$ & $(1,3)$ \\
\hline & & & & & 1 & 2 & 3 & $r$ & 1 & $(4,2)$ & $(0,2)$ & $(4,0)$ & $(4,0)$ \\
\hline
\end{tabular}

Table 11: The correspondence $(r, s) \mapsto(\ell, m)$ between the construction labels $(r, s)$ of the integrable boundary conditions on the right and the parafermionic conformal fields $(\ell, m)$ for the $A_{L}$ models with $L=3,4,5$ and number of faces $N=1 \bmod 2 k$.

\begin{tabular}{|c|c|c|c|}
\hline$(a, 0)$ & $(a, 2)$ & $(a, 2)$ & $(a, 0)$ \\
\hline$(O, 3)$ & $(O, 1)$ & $(O, 1)$ & $(O, 3)$ \\
\hline$(A, 0)$ & $(A, 2)$ & $(A, 2)$ & $(A, 0)$ \\
\hline 1 & 2 & 3 & 4 \\
\hline
\end{tabular}

$a \in D_{4}$
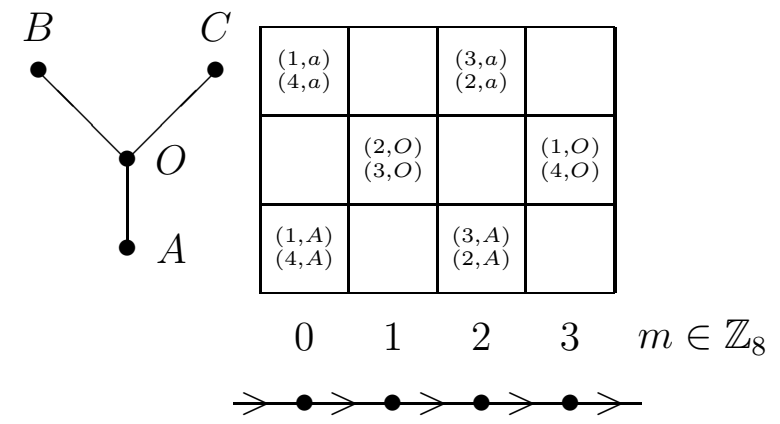

Table 12: The correspondence $(r, a) \mapsto(a, m)$ between integrable boundary conditions $(r, a)$ and conformal fields $(a, m)$ for the $\left(D_{4}, \mathbb{Z}_{8}\right)$ model with the number of faces $N=0 \bmod 4$. Within these tables $a=B$ or $C$. The minimal model Kac table symmetry reduces to the left/right flip in the $A_{4} \times D_{4}$ table.
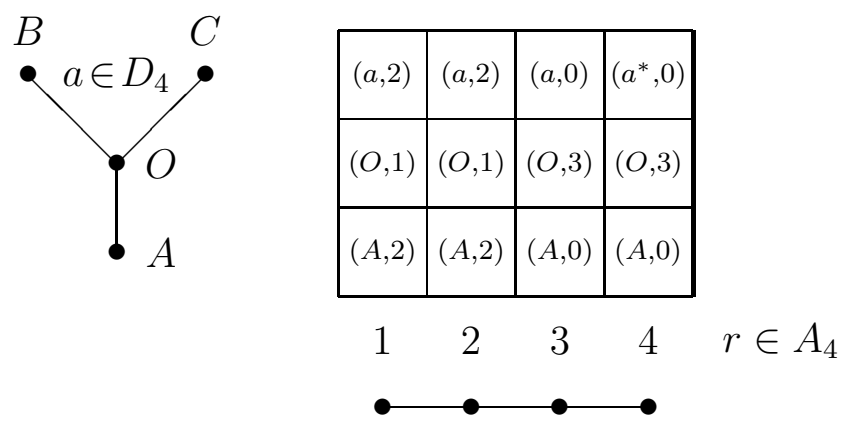

Table 13: The correspondence $(r, a) \mapsto(a, m)$ between integrable boundary conditions $(r, a)$ and conformal fields $(a, m)$ for the $\left(D_{4}, \mathbb{Z}_{8}\right)$ model with the number of faces $N=1 \bmod 4$. Note that $B^{*}=C$ and $C^{*}=B$. 
Comparing our numerical results with Table 8 allows us to obtain the correspondence given in Table 12 for the $\left(D_{4}, \mathbb{Z}_{8}\right)$ case with the number of faces $N=0 \bmod 4$ :

$$
\begin{gathered}
A_{4} \times D_{4} \\
(r, a)
\end{gathered} \quad \mapsto\left\{\begin{aligned}
D_{4} \times \mathbb{Z}_{8} & \\
(a, r-1) & \text { if } r+a \text { is odd } \\
(a, 4-r) & \text { if } r+a \text { is even }
\end{aligned}\right.
$$

For example, the partition function for the boundary conditions $(r, a)=(1, O)$ on the left and right is:

$$
\begin{aligned}
Z_{1 O \mid 1 O}^{D_{4}}(q) & =\sum_{s=1}^{5} V_{s} O \\
& =Z_{11 \mid 1 s}^{A_{5}}(q) \\
& Z_{11 \mid 11}^{A_{5}}(q)+2 Z_{11 \mid 13}^{A_{5}}(q)+Z_{11 \mid 15}^{A_{5}}(q)=\chi_{(0,0)}(q)+2 \chi_{(2,0)}(q)+\chi_{(4,0)}(q) .
\end{aligned}
$$

This result agrees with the alternative way of computing this partition function using the correspondence (4.5) and the extended fusion algebra described in Table 8:

$$
\begin{aligned}
Z_{1 O \mid 1 O}^{D_{4}}(q) & =Z_{(O, 3) \mid(O, 3)}^{D_{4}}(q) \\
& =\hat{\chi}_{(A, 0)}(q)+\hat{\chi}_{(B, 0)}(q)+\hat{\chi}_{(C, 0)}(q)=\chi_{(0,0)}(q)+\chi_{(4,0)}(q)+\chi_{(2,0)}(q)+\chi_{(2,0)}(q) .
\end{aligned}
$$

More generally, the correspondence in the $D$ and $E$ cases relate (2.19) and (4.4) as two compatible ways of computing partition functions in terms of $A_{L}$ partition functions.

As in the $A_{L}$ case, the table for $N=1 \bmod 4$ is obtained by a shifting procedure on the table, namely, shift the columns of the table to the left and interchange the $B$ and $C$ entries in the last column (see Table 13).

Using the intertwiner definition (4.4) and the correspondence formula (4.3), one finds that this result holds for any $D_{\frac{k}{2}+2}$ and for the exceptional cases $E_{6}, E_{7}$ and $E_{8}$ :

$$
(a, m)= \begin{cases}(a, r-1) & \text { if } r+a \text { is odd } \\ (a, k-r) & \text { if } r+a \text { is even }\end{cases}
$$

where $a \in G, r \in A_{k}$ and $m \in \mathbb{Z}_{2 k}$. This formula also holds for $G=A_{k+1}$ provided the ranges of $r$ and $s$ are adjusted to begin at 1 rather than 0 . Here the labelling of the nodes of the $D$ and exceptional $E$ graphs is as follows:

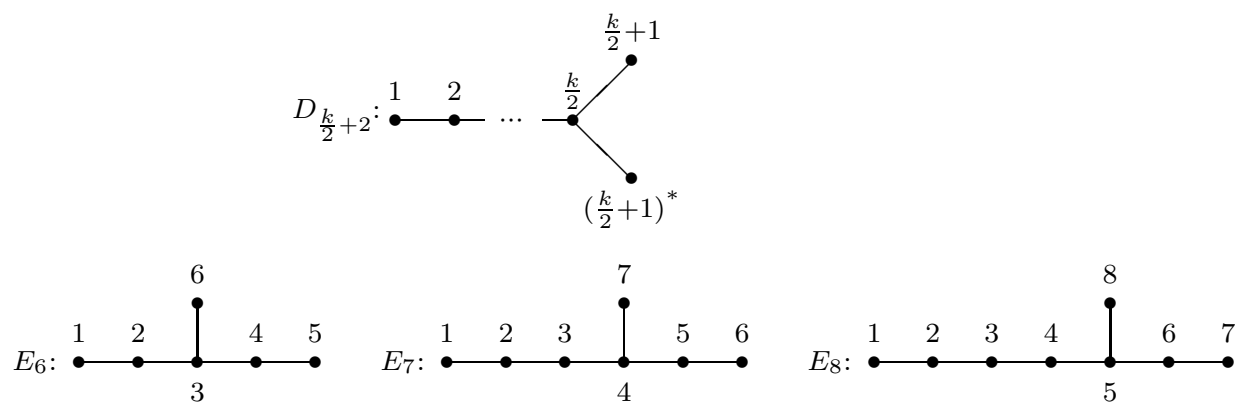




\section{$5 \quad$ Numerical Conformal Spectra}

In this section we describe our numerical analysis which confirm the results presented in Section 1 . As explained in the previous section, the spectra for the $D$ and $E$ models are related to the spectra of the $A$ models by intertwiners (4.4) so we need only do numerics on the $A$ parafermion models. The Yang-Baxter equation, boundary Yang-Baxter equation, reflection symmetry, inversion relation, crossing symmetry, height reversal symmetry and commutation of double row transfer matrices for various number of faces and all boundary conditions were checked numerically. The numerics were carried out for finite size row transfer matrices and extrapolated to large sizes. The spectra were obtained by numerical diagonalization of the row transfer matrices. For the $A_{L}$ lattice models the maximum number of faces $N$ in a row that we found manageable due to machine limitations was 22, 18 and 16 respectively for $L=3,4,5$.

We first studied the boundary conditions which from (4.2) should yield single characters, such as $(r, 1 \mid 1,1)$ or $(1, s \mid 1,1)$ with the number of faces $N=0 \bmod 2 k$. We computed numerically the double row transfer matrices for these boundary conditions for increasing $N$ and numerically diagonalized them to obtain the spectra. Allowing for the contribution of the bulk and boundary free energies, the central charge and the geometric factor, we fit the data corresponding to the largest eigenvalues of these transfer matrices, in negative integers powers of $N$ to extract the conformal weight $\Delta$. This value was compared with the entries in the parafermion Kac table to determine (up to conjugacy) the primary field associated with the given boundary condition.

In the $A_{5}$ case the level is $k=4$ and machine limitations prevent us from dealing with more than $N=16$ faces. Consequently, this direct method would give us only two numbers, for $N=8$ and $N=16$ faces respectively. However, some symmetries can be used to obtain more data for extrapolation to large $N$ by merging sequences for related boundary conditions and other mod properties as we now explain. Firstly, height reversal symmetry allows us to merge the sequence $(r, s \mid 1,1)$ for $N=0 \bmod 2 k$ with the sequence $(r, s \mid 1, L)$ for $N=k$ mod $2 k$. More generally, other sequences converge to the same character, namely $(r, s \mid m, 1)$ for $N=2 k-m \bmod 2 k$ and $(r, s \mid m, L)$ for $N=k-m \bmod 2 k$. Indeed, these boundary conditions are all compatible with the sawtooth shaped ground state. We discovered that this shift and flip procedure not only applies for the vacuum but for any boundary condition (see Table 11). This method of interleaving sequences allowed us to improve dramatically the accuracy of our numerics. A typical extrapolated numerical estimate of a conformal weight agrees to 5 or 6 digit accuracy with the exact entry in the parafermion Kac table.

In addition to producing the correct conformal weight $\Delta$, the numerical spectra should, in the case of a single character, also reproduce the correct degeneracies of the associated string function $\chi_{(\ell, m)}$. This means that if the eigenvalues $\Lambda_{n}^{(N)}$ of the double row transfer matrix with $N$ faces are placed in decreasing order with $n=0,1,2, \ldots$ then

$$
\Lambda_{n}^{(N)}=\Lambda_{0}^{(N)} \exp \left(-\frac{2 \pi \sin \vartheta}{N} a_{n}+o\left(\frac{1}{N}\right)\right)
$$




\begin{tabular}{c|cccccccccc} 
Energy & 1 & 2 & 3 & 4 & 5 & 6 & 7 & 8 & 9 & 10 \\
\hline Numerical & $1.04 \cdot 10^{-6}$ & 0.33331 & 0.999502 & 1.33363 & 1.33304 & 1.99795 & 2.01268 & 2.35139 & 2.33541 & 2.3362 \\
Exact & 0 & $\frac{1}{3}$ & 1 & $\frac{4}{3}$ & $\frac{4}{3}$ & 2 & 2 & $\frac{7}{3}$ & $\frac{7}{3}$ & $\frac{7}{3}$
\end{tabular}

Table 14: Comparison of the exact values and numerical estimates of the first ten energy levels for the $A_{5}$ parafermion model with $(1,3 \mid 1,3)$ boundary conditions. The cylinder partition function is $Z_{13 \mid 13}(q)=Z_{(2,0) \mid(2,0)}(q)=\chi_{(0,0)}(q)+\chi_{(2,0)}(q)+\chi_{(4,0)}(q)$.

where $a_{n}$ is an increasing integer sequence encoding the degeneracies in the character in terms of the modular parameter $q=\exp \left(-\frac{2 \pi}{N} \sin \vartheta\right)$. The leading corrections in the $o\left(\frac{1}{N}\right)$ term are negative integer powers of $N$ so a plot of the normalized log value against $1 / N$ becomes a straight line near zero and a polynomial fit in $1 / N$ gives a good extrapolation to $N=\infty$.

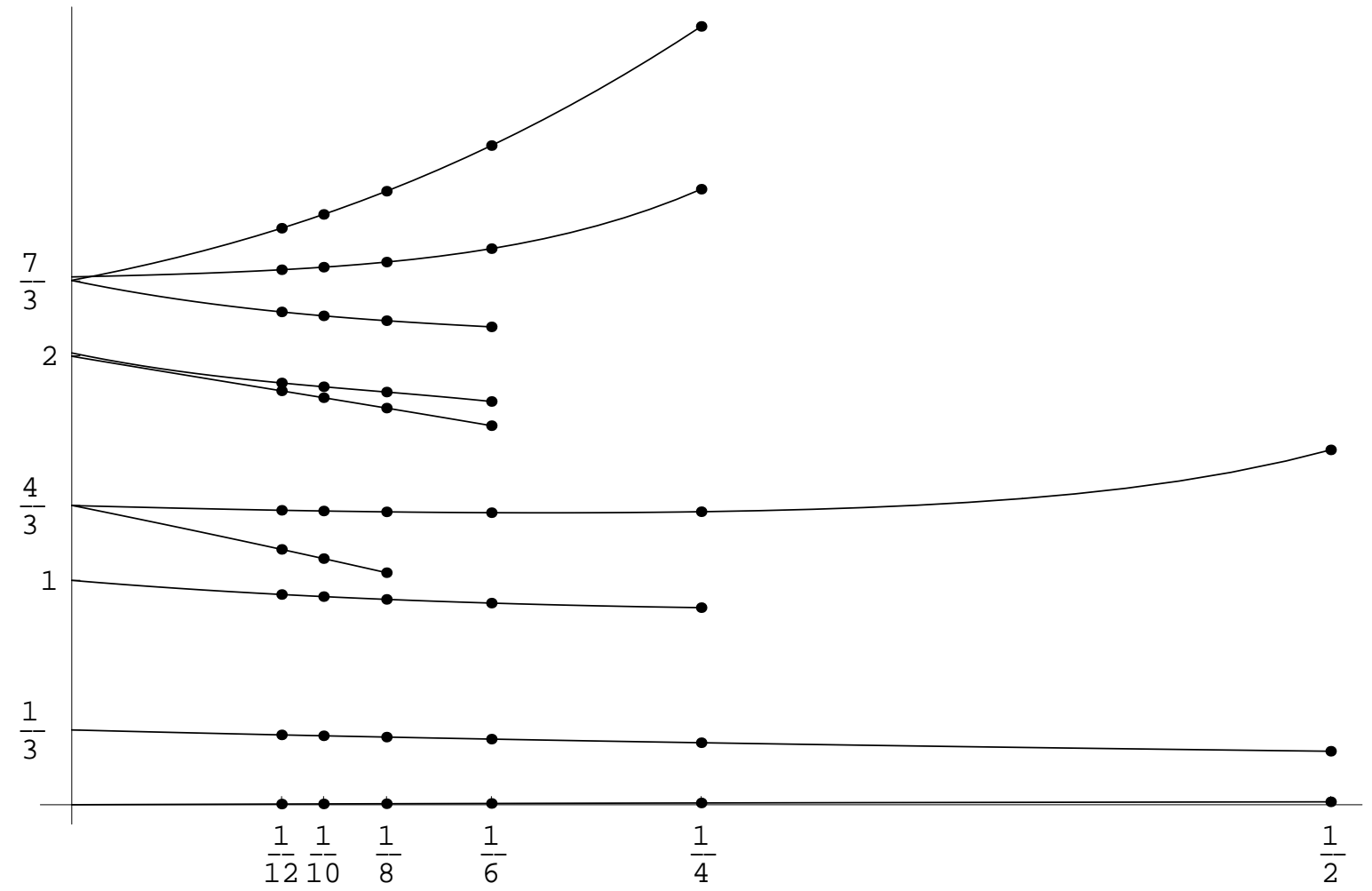

Figure 1: The extrapolated sequences corresponding to the first ten energy levels of the double row transfer matrix for the $A_{5}$ parafermion model with $(1,3 \mid 1,3)$ boundary conditions. The horizontal axis is $1 / N$ where $N$ is the number of faces.

After investigating all the boundary conditions leading to a single character we turned to the other boundary conditions and compared the numerical spectra with the predictions of the parafermion fusion algebra. In these cases the cylinder partition functions are sums of characters. As a typical example, consider the $\left(A_{5}, \mathbb{Z}_{8}\right)$ parafermion model with $(1,3 \mid 1,3)$ boundary conditions and $N=0 \bmod 8$. According to Table 10, the normalized partition 
function should converge to the sum of characters:

$$
\begin{aligned}
Z_{13 \mid 13}(q) & =Z_{(2,0) \mid(2,0)}(q)=\chi_{(0,0)}(q)+\chi_{(2,0)}(q)+\chi_{(4,0)}(q) \\
& =q^{-1 / 24}\left(1+q^{1 / 3}+q+2 q^{4 / 3}+2 q^{2}+3 q^{7 / 3}+5 q^{3}+o\left(q^{3}\right)\right)
\end{aligned}
$$

so the energy levels and their degeneracies should be given by the sequence

$$
\left\{a_{n}\right\}=\left\{0, \frac{1}{3}, 1, \frac{4}{3}, \frac{4}{3}, 2,2, \frac{7}{3}, \frac{7}{3}, \frac{7}{3}, 3 \ldots\right\}
$$

Table 14 and Figure 1 show a comparison of the extrapolated sequences and the exact values. The accuracy in other cases is at least as good as this case. As can be seen in Figure [, in extrapolating the sequences, the eigenvalues cannot simply be ordered according to their values at a given $N$. However, a given eigenvalue can be uniquely identified and tracked for each $N$ by examining its pattern of zeros in the complex $u$-plane.

The $D$ and $E$ parafermion models can be studied numerically by the same direct numerical diagonalization techniques as the $A$-type models. However, in these cases, it is better to use the intertwiner relations (4.4) to relate [27] the spectra of the $D$ or $E$ model to the spectra of various sectors (or boundary conditions) of the associated $A$ model with the same Coxeter number. In this way, for example, the spectra of the $D_{4}$ model with given boundary conditions is related to sectors of the $A_{5}$ model. Although it is not necessary to directly diagonalize the double row transfer matrices of the $D$ and $E$ parafermion models we did diagonalize some cases as checks on the numerics.

\section{Discussion}

In this paper we have shown how to associate integrable lattice boundary conditions to each conformal boundary condition $(a, m)$ of the $A-D-E \hat{s} \ell(2)$ parafermion models. Moreover, we have shown how the conformal labels $(a, m)$ of the string functions are related to the labels $(r, a)$ that naturally arise in the construction of the integrable boundary conditions. In contrast to the unitary minimals models, we observe (3.12) that the symmetry between the left and right boundaries is broken for the parafermion models due to the presence of an intrinsic cyclic chirality. We note also that there does not exist a gauge in which the local Boltzmann weights of the parafermion lattice models are all positive. Since the local weights should represent probabilities, this means that these models are not well defined as lattice models in statistical mechanics. Nevertheless, as is also the case for the non-unitary minimal models, these models yield well-defined conformal field theories in the continuum scaling limit.

In this work we have focussed on the principal parafermion models. It would be of interest to extend our results to the non-principal models. We expect these models to correspond to fused versions [21, 22] of the lattice models studied here. 


\section{Acknowledgements}

This research is supported by the Australian Research Council. We thank Roger Behrend for his careful reading and comments.

\section{References}

[1] Roger E. Behrend, Paul A. Pearce, and Jean-Bernard Zuber. Integrable boundaries, conformal boundary conditions and $A-D-E$ fusion rules. J. Phys. A, 31(50):L763-L770, 1998.

[2] Roger E. Behrend, Paul A. Pearce, Valentina B. Petkova, and Jean-Bernard Zuber. On the classification of bulk and boundary conformal field theories. Phys. Lett. B, 444(1-2):163-166, 1998.

[3] Roger E. Behrend, Paul A. Pearce, Valentina B. Petkova, and Jean-Bernard Zuber. Boundary conditions in rational conformal field theories. Nuclear Phys. B, 579(3):707$773,2000$.

[4] Roger E. Behrend and Paul A. Pearce. Integrable and conformal boundary conditions for $\hat{s \ell}(2) A-D-E$ lattice models and unitary minimal conformal field theories. J. Statist. Phys., 2000.

[5] V.B. Petkova and J.-B. Zuber. BCFT: from the boundary to the bulk. hep-th, 0009219:1-7, 2000.

[6] V.B. Petkova and J.-B. Zuber. The many faces of ocneanu cells. Nuclear Phys. B, 603:449-496, 2001.

[7] G. Pradisi, A. Sagnotti, and Ya. S. Stanev. Completeness conditions for boundary operators in 2D conformal field theory. Phys. Lett. B, 381(1-3):97-104, 1996.

[8] A. Cappelli, C. Itzykson, and J. B. Zuber. The A-D-E classification of minimal and $A_{1}^{(1)}$ conformal invariant theories. Comm. Math. Phys., 113(1):1-26, 1987.

[9] Vincent Pasquier. Two-dimensional critical systems labelled by Dynkin diagrams. $N u$ clear Phys. B, 285(1):162-172, 1987.

[10] A. B. Zamolodchikov and V. A. Fateev. Nonlocal (parafermion) currents in twodimensional conformal quantum field theory and self-dual critical points in $Z_{N^{-}}$ symmetric statistical systems. Zh. Èksper. Teoret. Fiz., 89(2):380-399, 1985.

[11] Doron Gepner and Zongan Qiu. Modular invariant partition functions for parafermionic field theories. Nuclear Phys. B, 285(3):423-453, 1987. 
[12] Zongan Qiu. Conformal field theories and modular invariant partition functions for parafermion field theories. Nuclear Phys. B, 295(2 FS21):171-187, 1988.

[13] P. Degiovanni. z/Nz conformal field theories. Comm. Math. Phys., 127(1):71-99, 1990.

[14] P. Degiovanni. $\mathbf{z} / N \mathbf{z}$ conformal field theories. In Number theory and physics (Les Houches, 1989), pages 2-19. Springer, Berlin, 1990.

[15] Jacques Distler and Zongan Qiu. BRS cohomology and a Feigin-Fuchs representation of Kac-Moody and parafermionic theories. Nuclear Phys. B, 336(3):533-546, 1990.

[16] Omar Foda and Trevor A. Welsh. Melzer's identities revisited. In Recent developments in quantum affine algebras and related topics (Raleigh, NC, 1998), pages 207-234. Amer. Math. Soc., Providence, RI, 1999. math.QA 9811156.

[17] Doron Gepner. On new conformal field theories with affine fusion rules. Nuclear Phys. $B, 561(3): 467-472,1999$.

[18] Atsushi Nakayashiki and Yasuhiko Yamada. Crystalline spinon basis for RSOS models. Internat. J. Modern Phys. A, 11(2):395-408, 1996.

[19] M. Jimbo, H. Konno, O. Odake, Y. Pugai, and J. Shiraishi. Free Field for the ABF Models in Regime 11. J. Statist. Phys., 102(3-4):13-76, 2001.

[20] R. J. Baxter. Hard hexagons: exact solution. J. Phys. A, 13(3):L61-L70, 1980.

[21] Andreas Klümper and Paul A. Pearce. Conformal weights of RSOS lattice models and their fusion hierarchies. Physica A, 183(3):304-350, 1992.

[22] Roger E. Behrend, Paul A. Pearce, and David L. O'Brien. Interaction-round-a-face models with fixed boundary conditions: the ABF fusion hierarchy. J. Statist. Phys., 84(1-2):1-48, 1996.

[23] Erik Verlinde. Fusion rules and modular transformations in 2D conformal field theory. Nuclear Phys. B, 300(3):360-376, 1988.

[24] Rodney J. Baxter. Exactly solved models in statistical mechanics. Academic Press Inc. [Harcourt Brace Jovanovich Publishers], London, 1989. Reprint of the 1982 original.

[25] David L. O’Brien, Paul A. Pearce, and Roger E. Behrend. Surface free energies and surface critical behaviour of the ABF models with fixed boundaries. In Statistical models, Yang-Baxter equation and related topics, and Symmetry, statistical mechanical models and applications (Tianjin, 1995), pages 285-295. World Sci. Publishing, River Edge, NJ, 1996. 
[26] David L. O'Brien and Paul A. Pearce. Surface free energies, interfacial tensions and correlation lengths of the ABF models. J. Phys. A, 30(7):2353-2366, 1997.

[27] Paul A. Pearce and Yu Kui Zhou. Intertwiners and $A-D-E$ lattice models. Internat. J. Modern Phys. B, 7(20-21):3649-3705, 1993. Yang-Baxter equations in Paris (1992). 